\title{
Bioactive Phytochemicals: Efficient Synthesis of Optically Active Substituted Flav-3-enes and Flav-3-en-3-o-R Derivatives
}

\author{
Matthew Chilaka Achilonu, ${ }^{1}$ Moosa Mahmood Sedibe, ${ }^{2}$ and Karabo Shale ${ }^{1}$ \\ ${ }^{1}$ Faculty of Natural Sciences, Mangosuthu University of Technology, Umlazi, Durban, KwaZulu-Natal, South Africa \\ ${ }^{2}$ Department of Agriculture, Faculty of Health and Environmental Sciences, Central University of Technology, \\ Free State, 1 Park Road, Bloemfontein 9301, South Africa \\ Correspondence should be addressed to Matthew Chilaka Achilonu; achilonu.matthew@mut.ac.za
}

Received 21 December 2016; Accepted 7 March 2017; Published 18 May 2017

Academic Editor: Bin Yu

Copyright (C) 2017 Matthew Chilaka Achilonu et al. This is an open access article distributed under the Creative Commons Attribution License, which permits unrestricted use, distribution, and reproduction in any medium, provided the original work is properly cited.

\begin{abstract}
The structural core of flavene (2-phenyl-2H-chromene) is commonly found in plant flavonoids, which exhibit a wide range of biological activities and diverse pharmacological profiles (e.g., antioxidant and anticancer activities). Flavonoids have attracted significant interest in medicinal and synthetic chemistry. Substituted flav-3-ene 13 was exclusively synthesized by the stereoselective elimination of the $O$-mesyl moiety on C-3 of 5,7,3', $4^{\prime}$-tetramethoxyflavan-3-mesylate 12 with 1,8-diazabicyclo[5.4.0] undec-7-ene. The reaction of 5,7,3', $4^{\prime}$-tetramethoxyflavan-3-one 15 with ytterbium trifluoromethanesulfonate in methanol afforded a novel 3-Osubstituted flav-3-ene derivative $\left(3,5,7,3^{\prime}, 4^{\prime}\right.$-pentamethoxyflav-3-ene) 1 . The reduction of 4 -(1,3,5-trihydroxybenzene)-5,7,3', $4^{\prime}$ tetra-O-benzylflavan-3-one $\mathbf{1 9 b}$ with hydrogen afforded a new compound: 3-hydroxy-4-(1,3,5-trihydroxybenzene)-5,7,3', $4^{\prime}$ tetrahydroxyflavan-3-en-3-ol 21 in good yield (95\%), while the acetylation of 19a and 21 afforded the expected novel flav-3-en3-acetoxy derivatives 20 (92\%) and 22 (90\%), respectively.
\end{abstract}

\section{Introduction}

The structural core of flavene (e.g., flav-3-ene (1)) is composed of 2-phenyl-2H-chromene, which is commonly categorized as a subfamily of natural flavonoids; flavonoids exhibit a wide range of biological activities [1-9]. Flavenes (e.g., 1 and flav2 -ene 2) are colourless compounds, which produce differentcoloured anthocyanidins (flavylium salts) under different $\mathrm{pH}$. Anthocyanidins are red in an acidic solution, violet in a neutral solution, and blue in an alkaline solution [10]. Flavylium salts bearing free phenolic hydroxyl groups are extremely unstable and short-lived compounds [10,11], while the substituted salts are stable that served as one of the few early sources of flavenes [10]. Some flavylium salts bearing an OR group at position 3 afford 1 , while flavylium salts with no substituent at position 3 afforded 2 [12]. These salts also exhibit resonating carbonium-ion structures of types $\mathbf{3}$ and $\mathbf{4}$, and by hydrolysis, these salts afford colourless pseudobases of hydroxyflavene-type $\mathbf{5}$ and 7 . The unstable chromenol moiety of $\mathbf{5}$ is expected to stabilize itself by equilibration with cis-chalcone 6 (Scheme 1). Jurd [13] has reported that the decolourisation of flavylium salts is caused by electron deficiency; hence, flavylium salts are readily attacked by exposure to water and other nucleophiles.

Because of the medicinal importance of the core structural unit of flavenes, there has been significant interest in the development of methods for scaffold construction [3-9]. However, previous studies have revealed that the synthesis of a functionalized flavene skeleton is a challenging task [5]; hence, few methods have reported the efficient stereoselective synthesis of flavenes [14-21]. Clark-Lewis and Jemison [14] have reported the first synthesis of racemic $\mathbf{1}$ based on chalcone starting materials. Zaveri [15], Pelter and Stainton [16], Nay et al. [17], and Deodhar et al. [18] have independently utilized similar cyclization routes to synthesize 1. The reactions involve the reduction of the C-4 carbonyl group to a 4-hydroxyl group, followed by the elimination of water to yield racemic 1. Casiraghi and Casnati [19] have used cinnamaldehyde and alkyl phenoxymagnesium bromides as the starting material to obtain racemic $\mathbf{1}$. When 
<smiles>O=C(/C=C\c1ccccc1O)c1ccccc1</smiles>

6<smiles>[CH-]CCCCCCC</smiles><smiles>OC1(c2ccccc2)C=Cc2ccccc2O1</smiles>

5<smiles>C1=C[C@@H](c2ccccc2)Oc2ccccc21</smiles>

1<smiles>[AlH][V]</smiles><smiles>c1ccc(-c2cc3ccccc3o2)cc1</smiles>

2

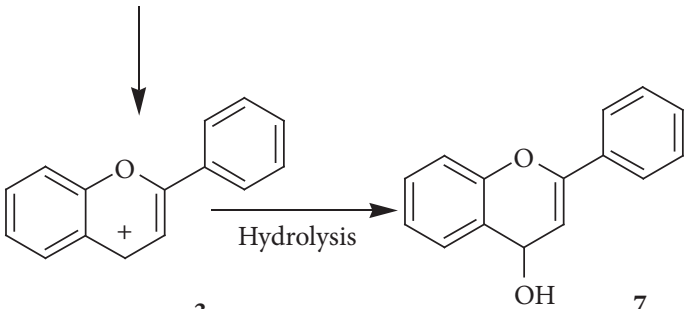

3

SCHEme 1: Resonating flavylium salt and flavene structural core.

$\mathbf{1}$ is heated under reflux in benzene in the presence of the corresponding phenoxymagnesium bromide, it isomerizes to 2. Cardillo et al. [20] have reported a biogenetic-like synthesis of racemic 1 from $o$-cinnamylphenols via the dehydrogenation of DDQ, while Subramanian and Balasubramanian [21] have synthesized racemic $\mathbf{1}$ from 1-arylprop-2-ynyl aryl ether in good yield by a facile Claisen rearrangement. Studies available on flavene synthesis have revealed that known reaction protocols afford racemic flavene products. Syntheses occurred via a cyclizable moiety, for example, chalcones and $o$-cinnamylphenol, or via the reduction of the C-4 carbonyl group and subsequent elimination of water, or the reduction of flavylium salts. To the best of our knowledge, no study has reported the elimination of the C-3 moiety flavan-3-ol to form 1. With this background, the substitution of the C3-OH of $(2 R, 3 S) 5,7,3^{\prime}, 4^{\prime}$-tetramethoxyflavan-3-ol (catechin) 8 and $(2 R, 3 R) 5,7,3^{\prime}, 4^{\prime}$-tetramethoxyflavan-3-ol (epicatechin) 9 with a good leaving group, such as a tosyl or mesyl group, has been hypothesized to synthesize a range of flavonoids and optically active substituted flav-3-enes. In addition, the use of substituted flavan-3-one for synthesizing substituted flav3-en-3-o-R substituted derivatives was considered. In this study, 5,7,3', $4^{\prime}$-tetramethoxyflav-3-ene 13 was synthesized in a facile, efficient manner by E2 elimination using a nonnucleophilic strong Lewis base, as well as 3-substituted flav-3-ene derivatives using a strong Lewis acid.

\section{Experimental}

2.1. Materials. Reagents and solvents, except THF, dichloromethane, and acetone, obtained from Merck, Fluka, and Sigma-Aldrich (Bloemfontein, South Africa), were used without further purification. THF was refluxed and distilled over sodium under inert gas with benzophenone as the indicator. Acetone was obtained by predrying commercial acetone with anhydrous $\mathrm{K}_{2} \mathrm{CO}_{3}$ for $24 \mathrm{~h}$ at room temperature. $\mathrm{K}_{2} \mathrm{CO}_{3}$ was filtered, and the solvent was distilled over $3 \AA$ molecular sieves and stored under $\mathrm{N}_{2}$. Dichloromethane was refluxed over $\mathrm{CaH}_{2}$ under nitrogen for $12 \mathrm{~h}$, followed by fresh distillation under nitrogen before use. Solid starting materials were dried in a vacuum oven at $60^{\circ} \mathrm{C}$ over $\mathrm{P}_{2} \mathrm{O}_{5}$ for $2-12 \mathrm{~h}$. Air- and moisture-sensitive reactions were performed under inert gas. Flash column chromatography and preparative liquid chromatography (PLC) were performed using Merck Kieselgel $60(0.063-0.20 \mathrm{~mm})$ silica gel with the indicated solvent or solvent mixture. Reaction progress was monitored with a Merck Kieselgel $60 \mathrm{PF}_{254}$ thin layer chromatography (TLC) sheet (aluminium back). A $2 \% \mathrm{v} / \mathrm{v}$ solution of formaldehyde (40\%) in concentrated $\mathrm{H}_{2} \mathrm{SO}_{4}$ was used as the spray reagent.

\subsection{Methods}

2.2.1. Characterization Techniques. Fourier transform infrared (FTIR) spectra were recorded on a Perkin-Elmer Spectrum One FTIR spectrometer by utilizing the $\mathrm{KBr}$ pellet technique. NMR spectra were recorded on a Bruker Avance spectrometer $(600 \mathrm{MHz})$ in $\mathrm{CDCl}_{3}(\delta 7.24 ; \delta 77.2)$, methanol- $d_{4}(\delta$ 4.87 and $3.31 ; \delta 49.2)$, or DMSO- $d_{6}(\delta 2.50 ; \delta 39.5)$. Chemical shifts were reported as parts per million (ppm) relative to trimethylsilane (TMS) as the internal standard. Coupling constants $(J)$ were measured in hertz. High-resolution electron ionization mass spectrometry (HREIMS) measurements were performed on a Micromass Q-TOF-2 spectrometer. Circular dichroism (CD) spectra were recorded on a Chirascan CD spectrometer.

\subsubsection{Standard Reaction Methods}

Purification of Catechin (Flavan-3-ol). Crude catechin was recrystallized by dissolving in boiling water, followed by the filtration of the hot solution. The filtrate was allowed to stand overnight for crystallization, and the solid was filtered and dried in a fume hood. The characterization data of the catechin agreed with catechin hydrate $([\alpha] / D+26 \pm 2)$ purchased from Sigma-Aldrich.

Acetylation of Samples. Acetylation was carried out by dissolving the dried phenolic material in a minimum volume of pyridine and acetic anhydride (twice the volume of pyridine used). After approximately $8-24 \mathrm{~h}$ at ambient temperature, the reaction was terminated by adding ice chips. Excess pyridine was removed from the precipitate by repetitive washing with cold water. 


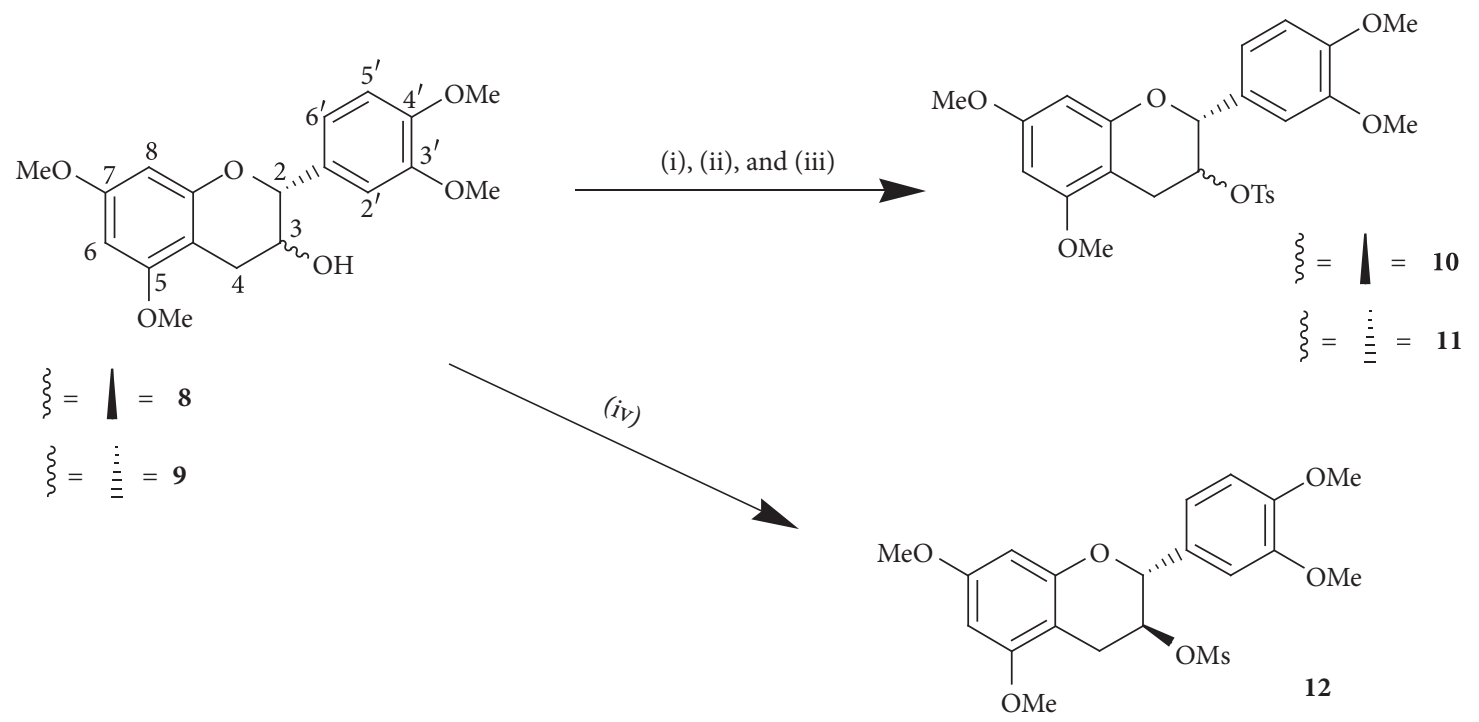

SCHEme 2: Mesylation and tosylation of flavan-3-ol. Reagents and conditions: (i) imidazole, THF, 1-toluenesulfonyl chloride, ice bath, (ii) step (i), methyl triflate, ice bath; (iii) 8 or $\mathbf{9}$, THF, N-methylimidazole, step (ii), rt, 24 h, $82 \%$; (iv) $8, \mathrm{MsCl}, \mathrm{DMAP}, \mathrm{THF}, \mathrm{Et} 3 \mathrm{~N}, \mathrm{rt}$ to $-5^{\circ} \mathrm{C}, 99 \%$.

Workup. Unless specified and appropriate for reaction workup, water was added to the reaction mixture, and the aqueous phase was extracted with diethyl ether or ethyl acetate. The organic extract was washed with water and dried with $\mathrm{Na}_{2} \mathrm{SO}_{4}$ or $\mathrm{MgSO}_{4}$, followed by the removal of solvent under reduced pressure at approximately $40^{\circ} \mathrm{C}$. Products were purified by TLC or flash column chromatography. Compounds 8, 9, and 5,7,3', $4^{\prime}$-tetra-methoxyflavan-3-one 15 were prepared according to previously reported procedures [22].

\subsection{Synthesis of Flav-3-enes and Derivatives}

Synthesis of 5,7,3',4'-Tetramethoxyflavan-3-ols (8, 9). First, $\mathrm{K}_{2} \mathrm{CO}_{3}$ (38 g, $276 \mathrm{mmol}$, dried at $240^{\circ} \mathrm{C}$ overnight) was added to an anhydrous acetone solution of predried commercial catechin or epicatechin $(10 \mathrm{~g}, 35 \mathrm{mmol})$ under nitrogen and stirred vigorously. Second, after $1 \mathrm{~h}$, dimethyl sulfate $\left(\left(\mathrm{CH}_{3}\right)_{2} \mathrm{SO}_{4}, 87 \mathrm{mg}, 276 \mathrm{mmol}\right)$ was slowly added over $30 \mathrm{~min}$ and refluxed for $2 \mathrm{~h}$. Third, at ambient temperature, $\mathrm{K}_{2} \mathrm{CO}_{3}$ was filtered, and acetone was removed under reduced pressure, followed by the neutralization of the excess $\left(\mathrm{CH}_{3}\right)_{2} \mathrm{SO}_{4}$ with cold ammonia $\left(80 \mathrm{~mL}, 25 \% \mathrm{NH}_{3} / \mathrm{H}_{2} \mathrm{O}, \mathrm{v} / \mathrm{v}\right)$. Next, the crude product was extracted with ethyl acetate $(2 \times$ $100 \mathrm{~mL})$, washed with water $(2 \times 70 \mathrm{~mL})$ and brine $(70 \mathrm{~mL})$, and dried over $\mathrm{MgSO}_{4}$, followed by solvent removal under reduced pressure. Finally, 8 or $\mathbf{9}$ was obtained as an offwhite, amorphous solid (99\%). The characterization of the compound was in agreement with reported data $[22,23]$.

Synthesis of 5,7,3', $4^{\prime}$-Tetramethoxyflavan-3-tosylates $(\mathbf{1 0}, \mathbf{1 1})$. The target product C-3-tosylated catechin 10 or C-3 tosylated epicatechin 11 is prepared in three steps. The first step involves the synthesis of 1-( $p$-toluenesulfonyl) imidazole: An anhydrous THF solution $(10 \mathrm{~mL})$ of imidazole $(3.6 \mathrm{~g}, 52.9 \mathrm{mmol})$ was stirred under nitrogen, followed by the dropwise addition of a THF $(8 \mathrm{~mL})$ solution of $p$-toluenesulfonyl chloride $(5 \mathrm{~g}, 26.2 \mathrm{mmol})$ over $10 \mathrm{~min}$. After $1 \mathrm{~h}$, the mixture was filtered and washed with THF, and the solvent was concentrated under reduced pressure to afford 1-( $p$-toluenesulfonyl) imidazole as white needle-like crystals. The crystals were washed with hexane and used in the next reaction step without further purification or characterization. The second step involves the preparation of the sulfonating agent. First, methyl triflate $(180 \mathrm{mg}, 1.10 \mathrm{mmol})$ was added dropwise to a $10 \mathrm{~mL}$ THF solution of 1 -( $p$-toluenesulfonyl) imidazole (266 mg, $1.20 \mathrm{mmol}$ ), which was cooled to $-5^{\circ} \mathrm{C}$ under nitrogen. Second, the reaction mixture was stirred at $-5^{\circ} \mathrm{C}$ for another hour to obtain the "sulfonating agent" for the third step of tosylation. For tosylation, methylated catechin $\mathbf{8}$ or methylated epicatechin 9 (346 mg, $1.0 \mathrm{mmol})$ was dissolved in dry THF $(10 \mathrm{~mL})$ under nitrogen, and $\mathrm{N}$-methylimidazole ( $82 \mathrm{mg}, 0.8 \mathrm{~mL}, 1.00 \mathrm{mmol}$ ) was added. The mixture was added to the off-white sulfonating reagent. After another $10 \mathrm{~min}$, the cooling system was removed, and the reaction continued at room temperature for $24 \mathrm{~h}$. The mixture was chromatographed on silica gel with 1:1 toluene-ethyl acetate as the solvent mixture for development, affording $\mathbf{1 0}$ or $\mathbf{1 1}$ as a white amorphous solid in $82 \%$ yield (Scheme 2 ). Physical data were in agreement with those reported previously [24].

Synthesis of 5,7,3',4'-Tetramethoxyflavan-3-mesylate (12). First, a mixture of oven-dried $8(346 \mathrm{mg}, 1.0 \mathrm{mmol})$ and 4-dimethylaminopyridine (DMAP) $(12.2 \mathrm{mg}, 0.1 \mathrm{mmol}$ ) was dissolved in dry THF $(5 \mathrm{~mL})$ under nitrogen. Triethylamine $(0.28 \mathrm{~mL}, 2.0 \mathrm{mmol})$ was added to a stirred solution and cooled to approximately $-5^{\circ} \mathrm{C}$ in a salt-ice bath for $10 \mathrm{~min}$ before methanesulfonyl chloride ( $172 \mathrm{mg}, 0.28 \mathrm{~mL}, 1.5 \mathrm{mmol})$ diluted with dry THF $(0.5 \mathrm{~mL})$ was added dropwise. After $30 \mathrm{~min}$, the reaction mixture was diluted with $10 \mathrm{~mL}$ ethyl acetate, washed with water $(2 \times 5 \mathrm{~mL})$ and $5 \mathrm{~mL}$ brine, and 
dried over anhydrous sodium sulfate. Ethyl acetate was removed under reduced pressure, and the crude product was purified with silica gel PLC (toluene-acetone $=9: 1$, $\mathrm{v} / \mathrm{v})$, affording the title compound $\mathbf{1 2}$ (99\% yield) as white needle-like crystals. ${ }^{1} \mathrm{H}$ NMR $\left(600 \mathrm{MHz}, \mathrm{CDCl}_{3}\right) \delta / \mathrm{ppm}$ : $6.91\left(\mathrm{dd}, J=1.7,8.2 \mathrm{~Hz}, 1 \mathrm{H}, \mathrm{H}-6^{\prime}\right), 6.90(\mathrm{~d}, J=1.7 \mathrm{~Hz}, 1 \mathrm{H}$, $\left.\mathrm{H}-2^{\prime}\right), 6.80\left(\mathrm{dd}, J=1.7,8.2 \mathrm{~Hz}, 1 \mathrm{H}, \mathrm{H}-5^{\prime}\right), 6.08(\mathrm{~d}, J=2.0 \mathrm{~Hz}$, $1 \mathrm{H}, \mathrm{H}-8), 6.04$ (d, $J=2.0 \mathrm{~Hz}, 1 \mathrm{H}, \mathrm{H}-6), 4.94$ (m, 1H, H-3), $4.90(\mathrm{~d}, J=7.7 \mathrm{~Hz}, 1 \mathrm{H}, \mathrm{H}-2), 3.09$ (ddd, $J=1.7,5.3,16.5 \mathrm{~Hz}$, $1 \mathrm{H}, \mathrm{H}-4 \alpha$ ), 2.85 (dd, $J=7.4,16.5 \mathrm{~Hz}, 1 \mathrm{H}, \mathrm{H}-4 \beta$ ), 2.43 (s, $\left.3 \mathrm{H}, \mathrm{OSO}_{2} \mathrm{CH}_{3}\right) .{ }^{13} \mathrm{C} \mathrm{NMR}\left(150 \mathrm{MHz}, \mathrm{CDCl}_{3}\right) \delta / \mathrm{ppm}: 160.2$ (C-5), $158.6(\mathrm{C}-7), 154.7$ (C-10), $149.6\left(\mathrm{C}-4^{\prime}\right), 149.4\left(\mathrm{C}-3^{\prime}\right)$, 129.8 (C-9), $119.8\left({\mathrm{C}-6^{\prime}}^{\prime}\right), 111.3\left(\mathrm{C}-2^{\prime}\right), 110.0\left(\mathrm{C}-5^{\prime}\right), 100.1(\mathrm{C}-1)$, 93.2 (C-8), 92.2 (C-6), 78.3 (C-2), 77.5 (C-3), 55.4-56.1 (4C, 4 $\left.\times \mathrm{OCH}_{3}\right), 38.1\left(\mathrm{C}, \mathrm{OSO}_{2} \mathrm{CH}_{3}\right), 26.5(\mathrm{C}-4) ; \mathrm{CD} \lambda \mathrm{nm}: 212.00$ $\left(2.200 \times 10^{4}\right), 227.00\left(-9.810 \times 10^{4}\right)$, and $234.00(1.190 \times$ $\left.10^{3}\right)$. HREIMS: $m / z 424\left(\mathrm{M}^{+}, 30\right), 328(100)$. Anal. calcd. for $\mathrm{C}_{20} \mathrm{H}_{24} \mathrm{O}_{8} \mathrm{~S}: \%$ C, 56.59; H, 5.70; O, 30.15; S, 7.55; found: \% C, 56.60; H, 5.67.

Synthesis of 5,7,3',4'-Tetramethoxyflavan-3-ene (13). First, DBU $(0.2 \mathrm{~mL}, 0.22 \mathrm{mmol})$ was added dropwise to an anhydrous acetonitrile solution $(1.5 \mathrm{~mL})$ of $12(30 \mathrm{mg}, 0.07 \mathrm{mmol})$ under $\mathrm{N}_{2}$, and the mixture was refluxed for $24 \mathrm{~h}$. Second, after cooling to ambient temperature, the reaction solvent was removed using a rotary evaporator. Next, the crude product was collected using ether $(10 \mathrm{~mL})$, followed by washing with water $(2 \times 5 \mathrm{~mL})$ and brine $(5 \mathrm{~mL})$. Finally, the organic phase was dried over $\mathrm{Na}_{2} \mathrm{SO}_{4}$, and the solvent was removed under reduced pressure to obtain $\mathbf{1 3}$ in $99 \%$ yield as a pink amorphous solid after alumina PLC (toluene-EtOAc $=8: 2$ ). ${ }^{1} \mathrm{H}$ NMR (600 MHz, $\mathrm{CDCl}_{3}$ ) $\delta / \mathrm{ppm}: 7.03(\mathrm{~d}, J=2.0 \mathrm{~Hz}, 1 \mathrm{H}$, $\left.\mathrm{H}-2^{\prime}\right), 7.01\left(\mathrm{dd}, J=2.0,8.1 \mathrm{~Hz}, 1 \mathrm{H}, \mathrm{H}-6^{\prime}\right), 6.86(\mathrm{~d}, J=8.1 \mathrm{~Hz}$, $\left.1 \mathrm{H}, \mathrm{H}-5^{\prime}\right), 6.83(\mathrm{dd}, J=2.0,10.0 \mathrm{~Hz}, 1 \mathrm{H}, \mathrm{H}-3), 6.06(\mathrm{~d}$, $J=2.2 \mathrm{~Hz}, 1 \mathrm{H}, \mathrm{H}-8), 6.04(\mathrm{~d}, J=2.2 \mathrm{~Hz}, 1 \mathrm{H}, \mathrm{H}-6), 5.79$ $(\mathrm{dd}, J=2.0,3.0 \mathrm{~Hz}, 1 \mathrm{H}, \mathrm{H}-2), 5.61(\mathrm{dd}, J=3.0,10.0 \mathrm{~Hz}, 1 \mathrm{H}$, $\mathrm{H}-4), 3.89-3.75\left(4 \mathrm{~s}, 12 \mathrm{H}, 4 \times \mathrm{OCH}_{3}\right) ;{ }^{13} \mathrm{C} \mathrm{NMR}(150 \mathrm{MHz}$, $\mathrm{CDCl}_{3}$ ) $\delta / \mathrm{ppm}: 161.3$ (C-7), 156.3 (C-5), 154.9 (C-10), 149.2 $\left(\mathrm{C}-3^{\prime}\right), 149.1\left(\mathrm{C}-4^{\prime}\right), 133.4\left(\mathrm{C}-1^{\prime}\right), 119.9(\mathrm{C}-4), 119.8\left(\mathrm{C}-6^{\prime}\right), 119.0$

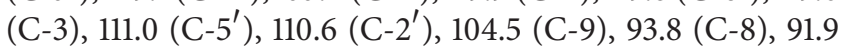
(C-6), $77.2(\mathrm{C}-2), 55.94-55.35\left(4 \mathrm{C}, 4 \times \mathrm{OCH}_{3}\right), \mathrm{CD} \lambda \mathrm{nm}(\theta)$ : $228.50\left(-2.356 \times 10^{3}\right)$. HREIMS: $m / z 328\left(\mathrm{M}^{+}, 100\right), 329((\mathrm{M}$ $\left.+\mathrm{H})^{+}, 20\right), 165$ (65). Anal. calcd. for $\mathrm{C}_{19} \mathrm{H}_{20} \mathrm{O}_{5}$ : \% C, 69.50; $\mathrm{H}, 6.14 ; \mathrm{O}, 24.36$; found: \% C, 69.52; $\mathrm{H}, 6.11$.

Synthesis of 5,7,3',4'-Tetramethoxyflavan Mixture $(\mathbf{1 3}, \mathbf{1 4})$. First, LDA $(0.5 \mathrm{~mL})$ was added to anhydrous THF $(5 \mathrm{~mL})$ at $-5^{\circ} \mathrm{C}$ under inert gas. After $10 \mathrm{~min}, 11$ (120 mg, $\left.0.2400 \mathrm{mmol}\right)$ was added, the cooling system was removed, and the reaction was gradually warmed up to ambient temperature within an hour. Next, the reaction solvent was removed under reduced pressure, and the crude product was purified by silica gel PLC $\left(\mathrm{CH}_{2} \mathrm{Cl}_{2}-\mathrm{n}\right.$-hexane-EtOAc $\left.=2: 8: 1, \times 2\right)$ to obtain a mixture of flavenes $\mathbf{1 3}$ and $\mathbf{1 4}$ as an orange paste in yields of $20 \%$ and $24 \%$, respectively.

5,7,3', $4^{\prime}$-Tetramethoxyflav-2-ene (14). ${ }^{1} \mathrm{H}$ NMR $(600 \mathrm{MHz}$, $\mathrm{CDCl}_{3}$ ) $\delta / \mathrm{ppm}: 7.24\left(\mathrm{dd}, J=2.0,8.4 \mathrm{~Hz}, 1 \mathrm{H}, \mathrm{H}-6^{\prime}\right), 7.23(\mathrm{~d}$, $\left.J=2.0 \mathrm{~Hz}, 1 \mathrm{H}, \mathrm{H}-2^{\prime}\right), 6.87\left(\mathrm{~d}, J=8.4 \mathrm{~Hz}, 1 \mathrm{H}, \mathrm{H}-5^{\prime}\right), 6.21$ (d, $J=2.3 \mathrm{~Hz}, 1 \mathrm{H}, \mathrm{H}-8), 6.15(\mathrm{~d}, J=2.3 \mathrm{~Hz}, 1 \mathrm{H}, \mathrm{H}-6), 5.40$ (d, $J=3.9 \mathrm{~Hz}, 1 \mathrm{H}, \mathrm{H}-3), 3.95-3.79\left(4 \mathrm{~s}, 12 \mathrm{H}, 4 \times \mathrm{OCH}_{3}\right), 3.3$ (d, $J=3.9 \mathrm{~Hz}, 2 \mathrm{H}, \mathrm{H}-4) .{ }^{13} \mathrm{C} \mathrm{NMR}\left(150 \mathrm{MHz}, \mathrm{CDCl}_{3}\right) \delta / \mathrm{ppm}$ : 159.5 (C-5), 158.1 (C-7), 152.8 (C-10), 149.1 (C-2), 148.6 (C-3'), $148.1\left(\mathrm{C}-4^{\prime}\right), 127.6\left(\mathrm{C}-1^{\prime}\right), 117.2\left(\mathrm{C}-6^{\prime}\right), 110.7\left(\mathrm{C}-5^{\prime}\right), 107.7(\mathrm{C}-$ $2^{\prime}$ ), 101.2 (C-9), 95.7 (C-3), 93.3 (C-6), 93.0 (C-8), 55.5-55.9 $\left(4 \mathrm{C}, 4 \times \mathrm{OCH}_{3}\right), 19.5(\mathrm{C}-4)$; HREIMS: $m / z 328\left(\mathrm{M}^{+}, 100\right), 165$ (65). Anal. calcd. for $\mathrm{C}_{19} \mathrm{H}_{20} \mathrm{O}_{5}$ : \% C, 69.50; H, 6.14; O, 24.36; found: \% C, 69.52; H, 6.11 .

Synthesis of 5,7,3', $4^{\prime}$-Tetramethoxyflavan-3-one (15). First, the Dess-Martin periodinane (DMP) $(7.5 \mathrm{~mL}, 0.3 \mathrm{M}$ solution of DMP in $\mathrm{CH}_{2} \mathrm{Cl}_{2}$ ) was added to a $\mathrm{CH}_{2} \mathrm{Cl}_{2}(5 \mathrm{~mL})$ solution of 8 (560 mg, $1.6 \mathrm{mmol}$ ) under nitrogen. Second, after stirring for $5 \mathrm{~min}, \mathrm{CH}_{2} \mathrm{Cl}_{2}(20 \mathrm{~mL})$ moistened with $0.2 \mathrm{~mL}$ water was added dropwise over $45 \mathrm{~min}$, resulting in a cloudy reaction mixture. The mixture was extracted with ether $(2 \times$ $30 \mathrm{~mL}$ ) and washed with a mixture of $10 \%$ sodium thiosulfate $\left(\mathrm{Na}_{2} \mathrm{~S}_{2} \mathrm{O}_{3}\right)$ and saturated $\mathrm{NaHCO}_{3}(2 \times 30 \mathrm{~mL}, 1: 1, \mathrm{v} / \mathrm{v})$, which separated the layers. The water phase was extracted with ether $(20 \mathrm{~mL})$, and the combined organic phases were washed with water and brine and dried over anhydrous sodium thiophosphate, followed by solvent removal under vacuum. The crude product was filtered over $\mathrm{SiO}_{2}(4 \times$ $4 \mathrm{~cm}$ glass column) with n-hexane-EtOAc $=6: 4$ and crystallized from the eluent, affording white needles in 95\% yield (Scheme 4). Spectral data were in agreement with those reported previously [20]. HREIMS: $m / z 344\left(\mathrm{M}^{+}, 100\right)$. Anal. calcd. for $\mathrm{C}_{19} \mathrm{H}_{20} \mathrm{O}_{6}$ : \% C, 66.27; $\mathrm{H}, 5.85 ; \mathrm{O}, 27.88$; found: $\%$ C, 66.32; H 5.65 .

Synthesis of 3,5,7,3',4'-Pentamethoxyflav-3-ene (17). First, the selected Lewis acid $\mathrm{Yb}(\mathrm{OTf})_{3}(200 \mathrm{mg}, 0.32 \mathrm{mmol})$ in methanol $(10 \mathrm{~mL})$ was added dropwise into a stirred THF $(5 \mathrm{~mL})$ solution of tetramethoxy-3-oxocatechin 15 (100 mg, $0.29 \mathrm{mmol}$ ) at $25^{\circ} \mathrm{C}$ under nitrogen. Second, the mixture was warmed at $40^{\circ} \mathrm{C}$ for approximately $6 \mathrm{~h}$. After workup and purification by alumina PLC (toluene-acetone $=9.9: 0.1$ ), two products $\mathbf{1 7}$ and $\mathbf{1 8}$ were obtained as off-white amorphous solids in yields of $17 \%$ and $32 \%$, respectively. ${ }^{1} \mathrm{H}$ NMR $\left(600 \mathrm{MHz} \mathrm{CDCl}_{3}\right) \delta / \mathrm{ppm}: 6.95\left(\mathrm{~d}, J=2.0 \mathrm{~Hz}, 1 \mathrm{H}, \mathrm{H}-2^{\prime}\right)$, $6.92\left(\mathrm{dd}, J=2.0,8.2 \mathrm{~Hz}, 1 \mathrm{H}, \mathrm{H}-6^{\prime}\right), 6.78(\mathrm{~d}, J=8.2 \mathrm{~Hz}, 1 \mathrm{H}$, H-5 $\left.{ }^{\prime}\right), 6.07$ (d, $\left.J=2.2 \mathrm{~Hz}, 1 \mathrm{H}, \mathrm{H}-8\right), 6.06(\mathrm{~d}, J=2.2 \mathrm{~Hz}, 1 \mathrm{H}$, H-6), 5.93 (s, 1H, H-4), 5.59 (s, 1H, H-2), 3.71-3.83 (5s, 15H, $\left.5 \times \mathrm{OCH}_{3}\right) ;{ }^{13} \mathrm{C} \mathrm{NMR}\left(150 \mathrm{MHz}, \mathrm{CDCl}_{3}\right) \delta / \mathrm{ppm}: 159.0(\mathrm{C}-7)$, 154.9 (C-5), 151.1 (C-10), 150.9 (C-3), $149.2\left(\mathrm{C}-3^{\prime}\right), 149.0\left(\mathrm{C}-4^{\prime}\right)$, $131.4\left(\mathrm{C}-1^{\prime}\right), 119.6\left(\mathrm{C}-6^{\prime}\right), 111.0\left(\mathrm{C}-5^{\prime}\right), 110.7\left(\mathrm{C}-2^{\prime}\right), 104.7(\mathrm{C}-9)$, 94.1 (C-8), 92.3 (C-6), 88.1 (C-4), 77.2 (C-2), 56.1-55.3 (5C, $5 \times$ $\left.\mathrm{OCH}_{3}\right)$; CD $\lambda \mathrm{nm}: 216.00\left(-2.566 \times 10^{4}\right)$. HREIMS: $m / z 358$ $\left(\mathrm{M}^{+}, 100\right), 359[\mathrm{M}+\mathrm{H}]^{+}, 343(10), 327$ (12); Anal. calcd. for $\mathrm{C}_{20} \mathrm{H}_{22} \mathrm{O}_{6}$ : \% C, 67.03; H, 6.19; O, 26.79; found: \% C, 67.03; $\mathrm{H}$ 6.20 .

3,3,5,7,3', $4^{\prime}$-Hexamethoxyflavane (18). ${ }^{1} \mathrm{H}$ NMR $(600 \mathrm{MHz}$, $\left.\mathrm{CDCl}_{3}\right) \delta / \mathrm{ppm}: 7.02\left(\mathrm{~d}, J=2.0 \mathrm{~Hz}, 1 \mathrm{H}, \mathrm{H}-2^{\prime}\right), 6.97(\mathrm{dd}$, $\left.J=2.0 \mathrm{~Hz}, 8.4 \mathrm{~Hz}, 1 \mathrm{H}, \mathrm{H}-6^{\prime}\right), 6.77\left(\mathrm{~d}, J=8.4 \mathrm{~Hz}, 1 \mathrm{H}, \mathrm{H}-5^{\prime}\right)$, $6.21(\mathrm{~d}, J=2.3 \mathrm{~Hz}, 1 \mathrm{H}, \mathrm{H}-8), 6.09$ (d, $J=2.3 \mathrm{~Hz}, 1 \mathrm{H}, \mathrm{H}-6)$, $5.19(\mathrm{~d}, J=2.0 \mathrm{~Hz}, 1 \mathrm{H}, \mathrm{H}-2), 3.85-3.32\left(6 \mathrm{~s}, 18 \mathrm{H}, 6 \times \mathrm{OCH}_{3}\right)$, $2.95(\mathrm{dd}, J=2.0,16.3 \mathrm{~Hz}, 1 \mathrm{H}, \mathrm{H}-4 \beta), 2.50(\mathrm{~d}, J=16.3 \mathrm{~Hz}, 1 \mathrm{H}$, 
$\mathrm{H}-4 \alpha) ;{ }^{13} \mathrm{C}$ NMR $\left(150 \mathrm{MHz}, \mathrm{CDCl}_{3}\right.$ ) $\delta /$ ppm: 159.9 (C-5), 158.3 (C-7), $154.6(\mathrm{C}-9), 148.5\left(\mathrm{C}-4^{\prime}\right), 148.3\left(\mathrm{C}-3^{\prime}\right), 130.7\left(\mathrm{C}-1^{\prime}\right), 119.7$ (C-6 $\left.{ }^{\prime}\right), 111.2\left(\mathrm{C}-2^{\prime}\right), 110.7\left(\mathrm{C}-5^{\prime}\right), 101.2(\mathrm{C}-3), 98.2(\mathrm{C}-10), 93.1$ (C-8), $91.6(\mathrm{C}-6), 76.8(\mathrm{C}-2), 48.6-55.8\left(6 \mathrm{C}, 6 \times \mathrm{OCH}_{3}\right), 25.9$ (C-4); CD: $\lambda \mathrm{nm}: 216.50\left(-3.312 \times 10^{2}\right)$. HREIMS: $m / z 390$ $\left(\mathrm{M}^{+}, 100\right), 391[\mathrm{M}+\mathrm{H}]^{+}, 345$ (8), 327 (25), 360 (22); Anal. calcd. for $\mathrm{C}_{21} \mathrm{H}_{26} \mathrm{O}_{9}$ : \% C, 64.60; $\mathrm{H}, 6.71 ; \mathrm{O} 28.69$; found: \% C, $64.55 ; \mathrm{H}, 6.68$.

Synthesis of 4-(1,3,5-Trihydroxybenzene)-5,7,3',4'-tetramethoxyflavan-3-one $(\mathbf{1 9 a})$. First, a stirred anhydrous THF mixture of 15 (50 mg, $0.145 \mathrm{mmol}$ ), phloroglucinol (151 mg, $1.2 \mathrm{mmol}$ ), and $\mathrm{AgBF}_{4}(215 \mathrm{mg}, 1.1 \mathrm{mmol})$ was refluxed for approximately $24 \mathrm{~h}$ under inert gas. Second, at ambient temperature, the reaction mixture was filtered through a wet silica gel pack using a n-hexane-ethyl acetate solvent mixture of $5: 5 \mathrm{v} / \mathrm{v}$, affording the crude product. The excess phloroglucinol was precipitated out using dichloromethane to obtain the required product 19a in good yield $(44.6 \mathrm{mg}$, $66 \%)$ as a light brown amorphous solid. IR $\left(\mathrm{KBr}, v / \mathrm{cm}^{-1}\right)$ : $1724(\mathrm{C}=\mathrm{O})$ and 1593 (aromatic $\mathrm{C}=\mathrm{C}$ stretch). ${ }^{1} \mathrm{H}$ NMR $\left(600 \mathrm{MHz}, \mathrm{CDCl}_{3}\right) \delta / \mathrm{ppm}: 7.11(\mathrm{dd}, J=8.1,10.0 \mathrm{~Hz}, 1 \mathrm{H}$, $\left.\mathrm{H}-6^{\prime}\right), 7.04\left(\mathrm{~d}, J=2.0 \mathrm{~Hz}, 1 \mathrm{H}, \mathrm{H}-2^{\prime}\right), 6.94(\mathrm{dd}, J=8.2$, $\left.10.0 \mathrm{~Hz}, 1 \mathrm{H}, \mathrm{H}-5^{\prime}\right), 6.20-6.10\left(\mathrm{~m}, 2 \mathrm{H}, \mathrm{H}-3^{\prime \prime} / 5^{\prime \prime}\right), 6.01$ (d, $J=2.2 \mathrm{~Hz}, 1 \mathrm{H}, \mathrm{H}-8), 5.96(\mathrm{~d}, J=2.2 \mathrm{~Hz}, 1 \mathrm{H}, \mathrm{H}-6), 5.10(\mathrm{~s}$, $1 \mathrm{H}, \mathrm{H}-2), 4.58$ (s, $1 \mathrm{H}, \mathrm{H}-4), 4.02-3.3 .79\left(\mathrm{~s}, 12 \mathrm{H}, 4 \times \mathrm{OCH}_{3}\right)$; ${ }^{13} \mathrm{C}$ NMR $\left(150 \mathrm{MHz}, \mathrm{CDCl}_{3}\right)$ 8/ppm: 160.1 (C-7), 160.0 $\left(\mathrm{C}-4^{\prime \prime}\right), 158.7\left(\mathrm{C}-2^{\prime \prime}\right), 158.4\left(\mathrm{C}-6^{\prime \prime}\right), 154.1(\mathrm{C}-10), 149.0\left(\mathrm{C}-4^{\prime}\right)$, $148.8\left(\mathrm{C}-3^{\prime}\right), 128.1\left(\mathrm{C}-1^{\prime}\right), 118.3\left(\mathrm{C}-1^{\prime \prime}\right), 111.3\left(\mathrm{C}-6^{\prime}\right), 111.1$ (C-5'), 109.4 (C-2'), 106.1 (C-9), 94.1 (C-8), 92.8 (C-6), 91.0 $\left(2 \mathrm{C}-3^{\prime \prime} / 5^{\prime \prime}\right), 82.8(\mathrm{C}-2), 55.2-55.9\left(4 \times \mathrm{OCH}_{3}\right), 38.0(\mathrm{C}-4)$; CD $\lambda \mathrm{nm}: 200.40\left(-8 \times 10^{3}\right), 237.20\left(3.221 \times 10^{4}\right)$. HREIMS: $m / z 343$ (100), $\mathrm{M}^{+}$468.4523, $(\mathrm{M}+\mathrm{H})^{+} 469$ (47). Anal. calcd. for $\mathrm{C}_{25} \mathrm{H}_{24} \mathrm{O}_{9}$ : \% C, 64.10; $\mathrm{H}, 5.16 ; \mathrm{O}, 30.74$; found: \% $\mathrm{C}$, 64.13; H, 5.14 .

Synthesis of 4-(1,3,5-Trihydroxybenzene)-5,7,3', $4^{\prime}$-tetra-O-benzylflavan-3-one (19b). First, a mixture of 1,3,5-hydroxybenzene $(1600 \mathrm{mg}, 4.04 \mathrm{mmol}), 5,7,3^{\prime}, 4^{\prime}$-tetra-O-benzylflavan3-one $15 \mathrm{~b}(600 \mathrm{mg}, 0.93 \mathrm{mmol})$, and $\mathrm{AgBF}_{4}(780 \mathrm{mg}$, $4.02 \mathrm{mmol}$ ) dried in a vacuum oven at $60^{\circ} \mathrm{C}$ for approximately $2 \mathrm{~h}$ was dissolved in anhydrous THF $(50 \mathrm{~mL})$ and refluxed under argon for $4 \mathrm{~h}$. Second, the reaction mixture was filtered over silica gel (n-hexane-EtOAc- $\mathrm{CHCl}_{3}, 8: 1: 1$ ) to remove the excess phloroglucinol and silver metal. The obtained crude product was purified by silica gel PLC chromatography (toluene-EtOAc, $10: 0.1$ ) to afford $19 \mathbf{b}$ as a brown amorphous solid (586 mg, 61\%). IR $\left(\mathrm{KBr}, v / \mathrm{cm}^{-1}\right)$ : 3032 (aromatic $\mathrm{C}-\mathrm{H}$ stretch), 1591 (aromatic $\mathrm{C}=\mathrm{C}$ bending), 1027 (C-O-C stretch). ${ }^{1} \mathrm{H}$ NMR $\left(600 \mathrm{MHz}\right.$, DMSO- $\left.d_{6}\right)$ $\delta /$ ppm: 7.40-7.09 (m, 20H, benzene-H), $6.96(\mathrm{~d}, J=8.3 \mathrm{~Hz}$, $\left.1 \mathrm{H}, \mathrm{H}-5^{\prime}\right), 6.93\left(\mathrm{~d}, J=2.0 \mathrm{~Hz}, 1 \mathrm{H}, \mathrm{H}-2^{\prime}\right), 6.71(\mathrm{dd}, J=2.0$, $\left.8.3 \mathrm{~Hz}, 1 \mathrm{H}, \mathrm{H}-6^{\prime}\right), 6.27\left(\mathrm{~s}, 2 \mathrm{H}, \mathrm{H}-3^{\prime \prime} / 5^{\prime \prime}\right), 6.26(\mathrm{~d}, J=2.3 \mathrm{~Hz}$, $1 \mathrm{H}, \mathrm{H}-8), 6.23$ (d, $J=2.3 \mathrm{~Hz}, 1 \mathrm{H}, \mathrm{H}-6), 5.33$ (s, $1 \mathrm{H}, \mathrm{H}-2)$, $5.24(\mathrm{~s}, 1 \mathrm{H}, \mathrm{H}-4), 5.07-4.70\left(\mathrm{~m}, 8 \mathrm{H}, 4 \times\right.$ benzylic- $\left.\mathrm{CH}_{2} \mathrm{O}\right)$. ${ }^{13} \mathrm{C}$ NMR $\left(150 \mathrm{MHz}, \mathrm{DMSO}-d_{6}\right) \delta / \mathrm{ppm}: 159.4-149.2$ (8C, aromatic-C-O), 137.8-137.2 (7C, aromatic quaternary-C), 128.8-127.6 (20C, benzene-C), 119.8 (C-6 $\left.{ }^{\prime}\right), 115.8\left(\mathrm{C}-5^{\prime}\right), 114.2$ (C-2'), 96.7 (C-6), $95.7(\mathrm{C}-8), 94.4\left(2 \mathrm{C}-3^{\prime \prime} / 5^{\prime \prime}\right), 82.5(\mathrm{C}-2)$,
71.5-70.1 (4C, $4 \times$ benzylic- $\left.\mathrm{CH}_{2} \mathrm{O}-\right)$ ), $39.3(\mathrm{C}-4)$. CD $\lambda \mathrm{nm}$ $(\theta): 220.00$ (17.695), 243.34 (8.465). HREIMS: $\mathrm{m} / z 772\left(\mathrm{M}^{+}\right.$, 25). Anal. calcd. for $\mathrm{C}_{49} \mathrm{H}_{40} \mathrm{O}_{9}$ : \% C, 76.15; H, 5.22; O, 18.63; found: \% C, 76.11; H 5.21.

Synthesis of 4-(1,3,5-Triacetoxybenzene)-5,7,3',4'-tetramethoxyflav-3-en-3-acetate (20). A light orange amorphous solid was obtained in $92 \%$ yield. ${ }^{1} \mathrm{H}$ NMR $\left(600 \mathrm{MHz}, \mathrm{CDCl}_{3}\right)$ $\delta /$ ppm: $7.07\left(\mathrm{t}, J=2.01,2.12,8.10 \mathrm{~Hz}, 2 \mathrm{H}, \mathrm{H}-2^{\prime} / 6^{\prime}\right), 6.92$ $\left(\mathrm{d}, J=2.25 \mathrm{~Hz}, 1 \mathrm{H}, \mathrm{H}-3^{\prime \prime}\right), 6.89(\mathrm{~d}, J=2.25 \mathrm{~Hz}, 1 \mathrm{H}, \mathrm{H}-$ $\left.6^{\prime \prime}\right), 6.84\left(\mathrm{~d}, J=8.10 \mathrm{~Hz}, 1 \mathrm{H}, \mathrm{H}-5^{\prime}\right), 6.18(\mathrm{~d}, J=2.32 \mathrm{~Hz}$, $1 \mathrm{H}, \mathrm{H}-8), 6.01(\mathrm{~d}, J=2.32 \mathrm{~Hz}, 1 \mathrm{H}, \mathrm{H}-6), 5.87$ (s, $1 \mathrm{H}, \mathrm{H}-2)$, $3.90-3.38\left(4 \mathrm{~s}, 12 \mathrm{H}, \mathrm{OCH}_{3}\right), 2.30-1.70\left(4 \mathrm{~s}, 12 \mathrm{H}, \mathrm{OCOCH}_{3}\right)$; ${ }^{13} \mathrm{C}$ NMR (150 MHz, $\mathrm{CDCl}_{3}$ ) $\delta / \mathrm{ppm}: 168.8,168.1-167.1$ (4C, aromatic methoxy-C), 160.0-153.1 (3C, aromatic quaternaryC), 148.5-147.5 (4C, aromatic acetoxy-C), 138.8, 127.7, 120.7, $118.9,112.5,112.4,112.1,110.9,109.5,103.6,93.0,92.2,76.7$, 54.9-54.3 (4C, $\left.\mathrm{OCH}_{3}\right)$ 20.1-19.1 (4C, $\left.\mathrm{OCOCH}_{3}\right)$; HREIMS: $m / z 636\left(\mathrm{M}^{+}, 100\right)$. Anal. calcd. for $\mathrm{C}_{33} \mathrm{H}_{32} \mathrm{O}_{13}: \% \mathrm{C}, 62.26$; H, 5.07; O, 32.67; found: \% C, 62.25; H 5.12.

Synthesis of 4-(1,3,5-Trihydroxybenzene)-5,7,3',4' -tetrahydroxyflav-3-en-3-ol (21). First, methanol $(4 \mathrm{~mL})$ was added to a stirred ethyl acetate $(4 \mathrm{~mL})$ solution of $19 \mathrm{~b}(66.9 \mathrm{mg}$, $0.064 \mathrm{mmol})$, followed by $\mathrm{Pd}(\mathrm{OH})_{2} / \mathrm{C}(33.5 \mathrm{mg})$, and purged with hydrogen for $2 \mathrm{~min}$. Second, after stirring under hydrogen at ambient temperature for another $1 \mathrm{~h}$, the mixture was filtered over cotton wool, and the solvent was removed under pressure, affording title compound $\mathbf{2 1}(25 \mathrm{mg}, 95 \%)$ as a dark brown solid: IR $\left(\mathrm{KBr}, v / \mathrm{cm}^{-1}\right): 3261$ (phenol OH, broad), 1603 (aromatic $\mathrm{C}=\mathrm{C}$ bending). ${ }^{1} \mathrm{H}$ NMR $\left(600 \mathrm{MHz}, \mathrm{MeOD}-d_{4}\right)$ $\delta /$ ppm: $6.82\left(\mathrm{~d}, 1 \mathrm{H}, J=2.0 \mathrm{~Hz}, \mathrm{H}-2^{\prime}\right), 6.73(\mathrm{~d}, 1 \mathrm{H}, J=8.2 \mathrm{~Hz}$, $\left.\mathrm{H}-5^{\prime}\right), 6.68\left(\mathrm{dd}, 1 \mathrm{H}, J=2.0,8.2 \mathrm{~Hz}, \mathrm{H}-6^{\prime}\right), 6.00(\mathrm{~d}, 1 \mathrm{H}, J=$ $2.2 \mathrm{~Hz}, \mathrm{H}-8), 5.78(\mathrm{~d}, 1 \mathrm{H}, J=2.2 \mathrm{~Hz}, \mathrm{H}-6), 5.80$ (s, broad, $\left.2 \mathrm{H}, \mathrm{H}-3^{\prime \prime} / 5^{\prime \prime}\right), 4.31$ (s, $\left.1 \mathrm{H}, \mathrm{H}-2\right) ;{ }^{13} \mathrm{C}$ NMR $(150 \mathrm{MHz}, \mathrm{MeOD}-$ $d_{4}$ ) $\delta /$ ppm: 157.5-155.8 (8C, aromatic-C-O), 137.8-137.2 (3C, aromatic quaternary $-\mathrm{C}), 118.4\left(\mathrm{C}-6^{\prime}\right), 114.7\left(\mathrm{C}-5^{\prime}\right), 113.2(\mathrm{C}-$ $\left.2^{\prime}\right), 104.8(\mathrm{C}-3), 101.6-95.3\left(2 \mathrm{C}-3^{\prime \prime} / 5^{\prime \prime}\right), 95.0$ (C-6), 94.2 (C-8), 78.6 (C-2), 39.3 (C-4); HREIMS: $m / z 412\left(\mathrm{M}^{+}, 100\right)$, found $\left[\mathrm{M}^{+}+\mathrm{H}\right]$ 413. Anal. calcd. for $\mathrm{C}_{21} \mathrm{H}_{16} \mathrm{O}_{9}: \% \mathrm{C}, 61.17 ; \mathrm{H}, 3.91$; $\mathrm{O}, 34.92$; found $\% \mathrm{C}, 61.19$; $\mathrm{H}, 3.81$.

Synthesis of 4-(1,3,5-Triacetoxybenzene)-5,7,3', $4^{\prime}$-tetraacetoxyflavan-3-en-3-acetate (22). A white amorphous solid was obtained in $90 \%$ yield. ${ }^{1} \mathrm{H}$ NMR $\left(600 \mathrm{MHz}, \mathrm{CDCl}_{3}\right) \delta / \mathrm{ppm}$ : $7.33\left(\mathrm{dd}, J=2.0,8.4 \mathrm{~Hz}, 1 \mathrm{H}, \mathrm{H}-6^{\prime}\right), 7.21(\mathrm{~d}, J=2.0 \mathrm{~Hz}, 1 \mathrm{H}, \mathrm{H}-$ $\left.2^{\prime}\right), 7.13\left(\mathrm{~d}, J=8.4 \mathrm{~Hz}, 1 \mathrm{H}, \mathrm{H}-5^{\prime}\right), 6.89(\mathrm{~d}, J=2.2 \mathrm{~Hz}, 1 \mathrm{H}, \mathrm{H}-8)$, $6.87(\mathrm{~d}, J=2.2 \mathrm{~Hz}, 1 \mathrm{H}, \mathrm{H}-6), 6.57\left(\mathrm{~d}, J=2.2 \mathrm{~Hz}, 2 \mathrm{H}, \mathrm{H}-3^{\prime \prime} / 5^{\prime \prime}\right)$, $5.91(\mathrm{~s}, 1 \mathrm{H}, \mathrm{H}-2), 2.22-1.61\left(8 \mathrm{~s}, 24 \mathrm{H}, \mathrm{OCOCH}_{3}\right) ;{ }^{13} \mathrm{C} \mathrm{NMR}$ $\left(150 \mathrm{MHz}, \mathrm{CDCl}_{3}\right) \delta / \mathrm{ppm}: 168.9-167.9$ (8C, aromatic-C-O) 153.3, $150.5\left(\mathrm{C}-1^{\prime \prime}\right), 150.2(\mathrm{C}-10), 149.2$, 149.1, $147.1\left(\mathrm{C}-1^{\prime}\right), 143.0$, $142.8,141.9,134.3,127.3\left(\mathrm{C}-6^{\prime}\right), 124.5\left(\mathrm{C}-2^{\prime}\right), 123.4\left(\mathrm{C}-5^{\prime}\right), 114.0$ (C-6), 113.7 (C-8), $112.5(\mathrm{C}-3), 110.8\left(\mathrm{C}-3^{\prime \prime}\right), 107.9\left(\mathrm{C}-5^{\prime \prime}\right), 76.9$ (C-2), 31.0, 29.2, 21.1-20.0 (8C, $\left.8 \times \mathrm{OCOCH}_{3}\right)$; HREIMS: $\mathrm{m} / z$ $748\left(\mathrm{M}^{+}, 100\right)$. Anal. calcd. for $\mathrm{C}_{37} \mathrm{H}_{32} \mathrm{O}_{17}: \% \mathrm{C}, 59.36 ; \mathrm{H}, 4.31$; O, 36.33; found: \% C, 60.5; $\mathrm{H}, 5.02$.

Synthesis of 4-(1,3,5-Trihydroxybenzene)-5,7,3', $4^{\prime}$-tetra-O-benzylflavan-3-ol (23). First, an aqueous $\mathrm{NaOH}$ solution 
$(0.15 \mathrm{~mL}, 2.0 \mathrm{M})$ was added to ethanol $(3 \mathrm{~mL})$, followed by $\mathrm{NaBH}_{4}(115.8 \mathrm{mg}, 3.06 \mathrm{mmol})$, and carefully dissolved. Second, to a stirring THF $(3 \mathrm{~mL})$ solution of $\mathbf{1 9 b}(110 \mathrm{mg}$, $0.11 \mathrm{mmol})$, methanol $(5 \mathrm{~mL})$ was added, followed by the slow addition of the previously prepared $\mathrm{NaBH}_{4}$ solution $(3 \mathrm{~mL})$. After approximately $30 \mathrm{~min}$, the reaction solvent was removed using a rotary evaporator and excess $\mathrm{NaBH}_{4}$ was neutralized with water $(1 \mathrm{~mL})$ and $10 \% \mathrm{HCl}(2 \mathrm{~mL})$. The organic phase was extracted, washed with $\mathrm{H}_{2} \mathrm{O}(\times 3)$ and brine, and dried over anhydrous $\mathrm{Na}_{2} \mathrm{SO}_{4}$, followed by solvent removal under pressure to obtain $(2 R, 4 S)-4-(1,3,5-$ trihydroxybenzene)-5,7,3', $4^{\prime}$,-tetra-O-benzylflavan-3-ol 23 (109 $\mathrm{mg}, 99 \%)$ as a light yellow paste. IR $\left(\mathrm{KBr}, v / \mathrm{cm}^{-1}\right)$ : 3031 (aromatic C-H stretch), 1603 (aromatic $\mathrm{C}=\mathrm{C}$ bending), 1591 (aromatic $\mathrm{C}=\mathrm{C}$ stretch), 1027 (C-O-C stretch, diaryl). ${ }^{1} \mathrm{H}$ NMR $\left(600 \mathrm{MHz}, \mathrm{DMSO}-d_{6}\right) \delta / \mathrm{ppm}$ : 7.45-7.15 (20H, m, benzene-H), $7.06\left(\mathrm{~d}, 1 \mathrm{H}, J=1.9 \mathrm{~Hz}, \mathrm{H}-2^{\prime}\right.$ ), 7.01 (s br., $2 \mathrm{H}$, $\left.\mathrm{H}-3^{\prime \prime} / 5^{\prime \prime}\right), 6.98\left(\mathrm{~d}, 1 \mathrm{H}, J=8.3 \mathrm{~Hz}, \mathrm{H}-5^{\prime}\right), 6.87$ (dd, $1 \mathrm{H}, J=$ 1.9, 8.3 Hz, H-6 $\left.{ }^{\prime}\right), 6.24$ (d, 1H, J = 2.3 Hz, H-8), 5.97 (d, 1H, $J$ $=2.3 \mathrm{~Hz}, \mathrm{H}-6), 5.11-4.75\left(8 \mathrm{H}, \mathrm{m}, 4 \times\right.$ benzylic $\left.-\mathrm{CH}_{2} \mathrm{O}\right), 4.85$ (d, $J=10.0 \mathrm{~Hz}, 1 \mathrm{H}, \mathrm{H}-2), 4.81(\mathrm{~d}, J=6.5 \mathrm{~Hz}, 1 \mathrm{H}, \mathrm{H}-4), 4.12$ (dd, $1 \mathrm{H}, J=6.2,8.8 \mathrm{~Hz}, \mathrm{H}-3$ ), 3.48 (s very br., $1 \mathrm{H}, \mathrm{OH}-3$ ). ${ }^{13} \mathrm{C}$ NMR (150 MHz, DMSO- $d_{6}$ ) $\delta / \mathrm{ppm}: 159.0-149.1$ (8C, aromatic-C-O), 138.1-137.7 (7C, aromatic quaternary-C), 128.8-127.5 (20C, benzene-C), $121.6\left(\mathrm{C}-6^{\prime}\right), 116.3\left(\mathrm{C}-2^{\prime}\right), 116.0$ $\left(\mathrm{C}-5^{\prime}\right), 96.1(\mathrm{C}-6), 95.9\left(2 \mathrm{C}-3^{\prime \prime} / 5^{\prime \prime}\right), 94.1$ (C-8), $78.6(\mathrm{C}-2)$, 71.9-70.2 (4C, $4 \times$ benzylic- $\mathrm{CH}_{2} \mathrm{O}$ ), 70.7 (C-3), 32.8 (C-4); CD $\lambda$ nm: 216.70 (22.695) and 245.01 (8.465). HREIMS: $m / z$ $774\left(\mathrm{M}^{+}, 100\right)$. Anal. calcd. for $\mathrm{C}_{49} \mathrm{H}_{42} \mathrm{O}_{9}$ : \% C, 75.95; H, 5.46; O, 18.58; found: \% C, 75.99; H 5.41.

Synthesis of 4-(1,3,5-Trihydroxybenzene)-5,7,3',4'-tetrahydroxyflavan-3-ol (24). First, methanol $(4 \mathrm{~mL})$ was added to a stirred THF $(4 \mathrm{~mL})$ solution of $23(100 \mathrm{mg}, 0.129 \mathrm{mmol})$, followed by $\mathrm{Pd}(\mathrm{OH})_{2} / \mathrm{C}(33.5 \mathrm{mg})$, and purged with hydrogen for $2 \mathrm{~min}$. Second, after $1 \mathrm{~h}$ under hydrogen at ambient temperature and pressure, the mixture was filtered over cotton wool, and the solvent was removed under pressure to obtain 24 (50 mg, 94\%) as a brown solid: IR (KBr, $\left.v / \mathrm{cm}^{-1}\right)$ 3261 (phenol OH, broad), 1603 (aromatic $\mathrm{C}=\mathrm{C}$ bending). ${ }^{1} \mathrm{H}$ NMR $\left(600 \mathrm{MHz}, \mathrm{MeOD}-d_{4}\right) \delta / \mathrm{ppm}: 6.82(\mathrm{~d}, J=2.0 \mathrm{~Hz}, 1 \mathrm{H}$, $\left.\mathrm{H}-2^{\prime}\right), 6.73\left(\mathrm{~d}, J=8.2 \mathrm{~Hz}, 1 \mathrm{H}, \mathrm{H}-5^{\prime}\right), 6.68(\mathrm{dd}, J=2.0,8.2 \mathrm{~Hz}$, $\left.1 \mathrm{H}, \mathrm{H}-6^{\prime}\right), 5.93$ (d, $\left.J=2.4 \mathrm{~Hz}, 1 \mathrm{H}, \mathrm{H}-6\right)$, and $5.84(\mathrm{~d}, J=2.4 \mathrm{~Hz}$, $1 \mathrm{H}, \mathrm{H}-8), 5.88$ (s, 2H, H-3"/ $\left.5^{\prime \prime}\right), 5.04$ (d, $J=7.2 \mathrm{~Hz}, 1 \mathrm{H}, \mathrm{H}-$ 2), $4.70(\mathrm{~d}, J=5.2 \mathrm{~Hz}, 1 \mathrm{H}, \mathrm{H}-4), 4.18(\mathrm{dd}, J=5.2,7.2 \mathrm{~Hz}$, $1 \mathrm{H}, \mathrm{H}-3) .{ }^{13} \mathrm{C}$ NMR $\left(150 \mathrm{MHz}, \mathrm{MeOD}-d_{4}\right) \delta / \mathrm{ppm}: 157.5-155.8$ (8C, aromatic-C-O), 132.7-131.1 (3C, aromatic quaternaryC), $118.4\left({\mathrm{C}-6^{\prime}}^{\prime}\right), 114.7\left(\mathrm{C}-5^{\prime}\right), 113.2\left(\mathrm{C}-2^{\prime}\right), 101.6\left(\mathrm{C}-3^{\prime \prime}\right), 95.3$ $\left(\mathrm{C}-5^{\prime \prime}\right), 95.0$ (C-6), 94.2 (C-8), 78.6 (C-2), 72.1 (C-3), 31.1 (C4). HREIMS: $m / z 414\left(\mathrm{M}^{+}, 100\right)$. Anal. calcd. for $\mathrm{C}_{21} \mathrm{H}_{18} \mathrm{O}_{9}$ : $\%$ C, 60.87; H, 4.38; O, 34.75; found \% C, 60.87; H 4.38.

\section{Results and Discussion}

Efforts to eliminate or replace the secondary hydroxyl group at the C-3 of flavan-3-ol with a carbon-carbon bond were seemingly elusive, and attempts resulted in the decomposition of substrates after several hours of reaction. The nonsubstitution or effective elimination of the carbon-3 moiety possibly occurred via the pathway of an antibonding orbital, which is "hidden" inside the heterocyclic C-ring (most likely in a chair conformation) rather than the $\mathrm{S}_{\mathrm{N}} 2$ reaction pathway; thus, it is not accessible to the approaching electronrich nucleophile [5] or by the nonnucleophilic strong base to abstract the $\mathrm{OH}$ proton by E2-type elimination.

By a simple method, a mixture of $\mathbf{8}$ and a mole equivalent of DMAP in THF under inert gas with methanesulfonyl chloride and excess triethylamine at subzero temperatures afforded 12 in excellent yield. After workup, the compound required no further purification before use in the elimination step. On the other hand, C-3-tosylated catechin 10 and C-3 tosylated epicatechin $\mathbf{1 1}$ were synthesized in three steps: (i) synthesis of 1-( $p$-toluenesulfonyl)imidazole, (ii) preparation of a sulfonating agent, and (iii) tosylation (Scheme 2).

The stereoselective elimination of the tosyl or the mesyl group at the C-3 of methylated catechin in anhydrous THF with a strong base at low temperature exclusively afforded flav-3-ene in low yields of 5\% and 15\%, respectively. By refluxing an anhydrous acetonitrile solution of catechin tosylate $\mathbf{1 0}$ or catechin mesylate $\mathbf{1 2}$ in the presence of DBU exclusively afforded 13 in good yields of $82 \%$ and $99 \%$, respectively (Scheme 3). The reaction with $\mathbf{1 1}$ was repeated by substituting acetonitrile with dry THF, followed by refluxing the mixture for $24 \mathrm{~h}$ to afford 13 in a very low yield of $7.5 \%$. This low yield is possibly caused by a radical inhibitor present in THF, which could retard the DBU activity. By refluxing the acetonitrile solution of $\mathbf{1 1}$ in the presence of DBU, a mixture of $\mathbf{1 3}$ and $14(80 \%$ combined yield) in a ratio of approximately $1: 1$ was obtained, while the addition of $\mathbf{1 1}$ to an anhydrous THF solution of lithium diisopropylamide (LDA) at approximately $-5^{\circ} \mathrm{C}$ also afforded $\mathbf{1 3}$ and $\mathbf{1 4}$ (44\% combined yield) as an orange paste (Scheme 3 ). The product mixture of flavenes from the elimination of $\mathbf{1 1}$ can be explained by the basecatalysed trans elimination to a flav-2-ene of a hydrogen at $\mathrm{C}-2$ trans to the tosyl group at $\mathrm{C}-3$. This was in contrast to $(2 R, 3 S)$ tetra-O-methyl-3-O-tosyl-catechin $\mathbf{1 0}$, where the hydrogen at position 2 was cis to the tosyl group at C-3, and the only available trans hydrogen was at $\mathrm{C}-4$, resulting in the exclusive formation of flav-3-ene and the retention of configuration at C-2. Typically, it was difficult to synthesize 2 and $\mathbf{1}$ because of their facile oxidation anthocyanidins.

The ${ }^{1} \mathrm{H}$ NMR spectra of the 3-O-derivatives $\mathbf{9}, \mathbf{1 0}$, and $\mathbf{1 1}$ clearly indicated the presence of an aromatic ABX system (dd, $J=2.0,8.0 \mathrm{~Hz} ; \mathrm{d}, J=2.0 \mathrm{~Hz}$ ) and an $\mathrm{AB}$ resonance system $(J=2.0 \mathrm{~Hz})$, representing the catechol and phloroglucinol character of the $\mathrm{B}$ and $\mathrm{A}$ rings, respectively. From the $\mathrm{CD}$ spectra of 9 and 11, the optical activities at C-2 and C3 were maintained. As expected, 13 exhibited three oneproton resonances for the $\mathrm{C}$-ring, a complex $\mathrm{ABX}$ system. The olefinic protons on $\mathrm{C}-3$ and $\mathrm{C}-4(\mathrm{H}-3$ and $\mathrm{H}-4)$ were coupled by cis-coupling $\left(J_{3,4}=10 \mathrm{~Hz}\right)$, and the proton on $\mathrm{C}-2(\mathrm{H}-2)$ was coupled to both $\mathrm{H}-3$ and $\mathrm{H}-4\left(J_{2,3}=3 \mathrm{~Hz}\right.$, and $\left.J_{2,4}=2 \mathrm{~Hz}\right)$. The benzylic proton $\mathrm{H}-2$ was adjacent to an ether oxygen, which was observed at $\delta 5.76$ as a doublet of doublets $(J=$ 2.0, 3.0 Hz). Flav-3-enes contained a chiral carbon on C-2. The HMBC correlation between C-2/C-5 and H-4 in HMBC permitted distinction between $\mathrm{H}-4$ and $\mathrm{H}-3$. The ${ }^{13} \mathrm{C}$ APT 


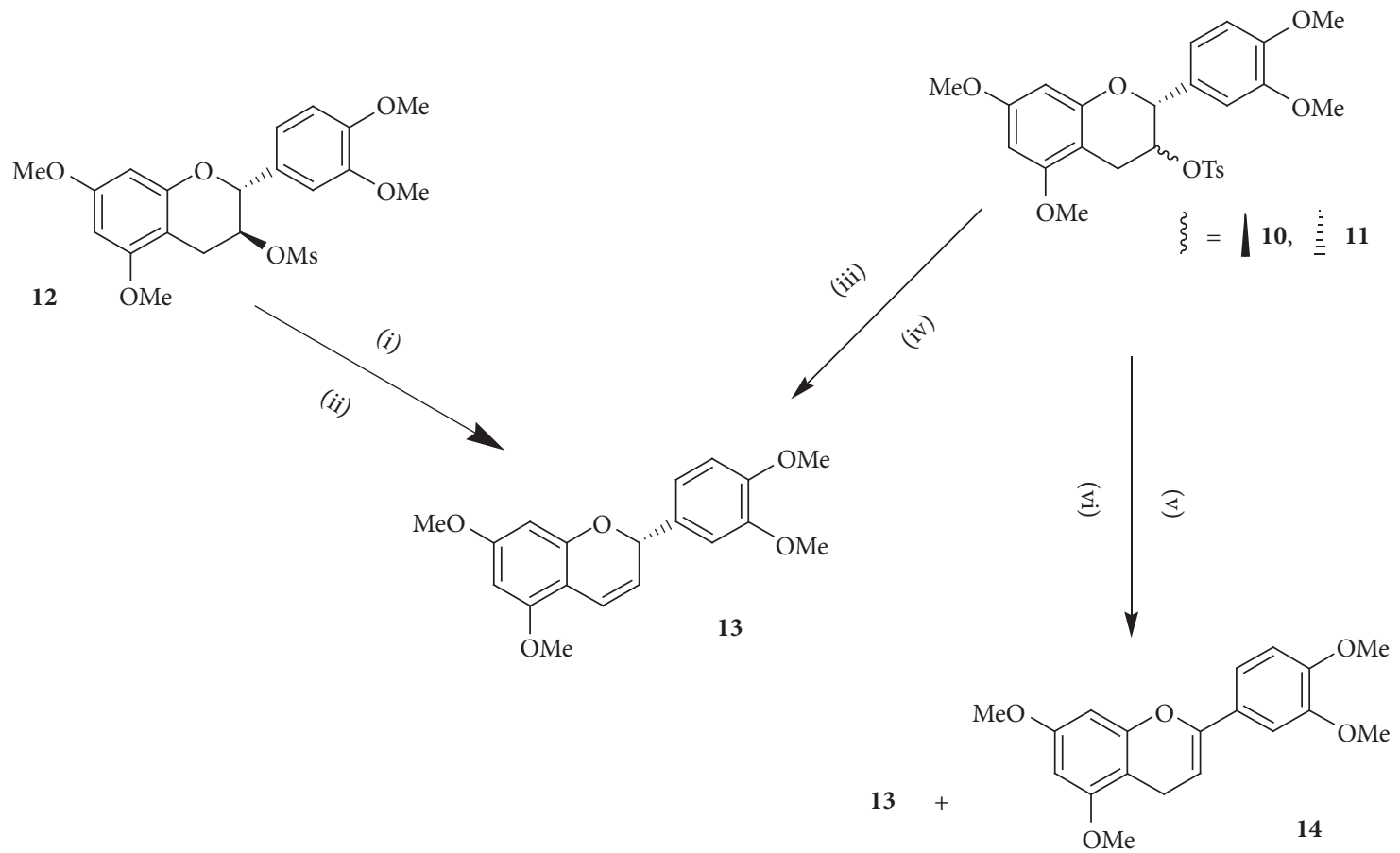

Scheme 3: Synthesis of flavenes. Reagents and conditions: (i) 12, LDA, THF, ice bath, 15\%; (ii) 12, DBU, MeCN, reflux, 99\%; (iii) 10, LDA, THF, ice bath, 5\%; (iv) 10, DBU, MeCN, reflux, 82\%; (v) 11, LDA, THF, ice bath, $44 \%$; (vi) 11, DBU, MeCN, reflux, $80 \%$.<smiles>[R]Oc1cc(O[R])c2c(c1)O[C@H](c1ccc(O)c(O[R1])c1)[C@@H](O)C2</smiles><smiles>[R10]Oc1cc(O[R1])c2c(c1)O[C@H](c1ccc(O[R1])c(O[R1])c1)C(=O)C2</smiles>

15 (ii)<smiles>[R10]Oc1cc(OC)c2c(c1)O[C@H](c1ccc(OC)c(OCC)c1)[C@@](C)(O)C2</smiles>

16<smiles>CCOc1ccc([C@@H]2Oc3cc(OC)cc(OC)c3C=C2OC)cc1O</smiles>

17<smiles>[R]Oc1cc(O)c2c(c1)O[C@H](c1ccc(OC)c(OC)c1)C(OC)(OC)C2</smiles>

18

Scheme 4: Synthesis of flav-3-en-3-o-R substituents. Reagents and conditions: (i) DMP, $\mathrm{CH}_{2} \mathrm{Cl}_{2}, \mathrm{H}_{2} \mathrm{O}, 95 \%$; (ii) $\mathrm{Yb}(\mathrm{OTf})_{3}$, MeOH, reflux, $49 \%$.

and HSQC spectra suggested that the signal of C-2 overlaps with that of chloroform at $\delta 77.2 \mathrm{ppm}$ in ${ }^{13} \mathrm{C}$ NMR spectrum. The HSQC correlation between $\mathrm{H}-4$ and C-4 permitted distinction between C-3 and C-4. Strong correlations were observed between protons $\mathrm{H}-2, \mathrm{H}-3$, and $\mathrm{H}-4$ in the COSY experiment, confirming the presence of the vinyl group in the C-ring. In addition, flav-3-ene clearly exhibited a negative Cotton effect at $\lambda=228 \mathrm{~nm}$. The observed retention of 
configuration at C-2 indicated that optically active flav-3-ene is successfully synthesized. Two proton signals corresponding to the C-ring were observed in 14: a two-proton doublet $\left(J_{4}=\right.$ $3.8 \mathrm{~Hz})$ on $\mathrm{C}-4\left(\mathrm{CH}_{2}\right.$ group) and a one-proton triplet bonded to $\mathrm{C}-3$ (H-3), observed at $\delta 5.40$. Both allylic and benzylic $\mathrm{CH}_{2}\left(\mathrm{H}-4_{\alpha / \beta}\right.$ and $\left.\mathrm{C}-4\right)$ groups were observed at $\delta 3.34$ and $19.49 \mathrm{ppm}$ in the ${ }^{1} \mathrm{H}$ NMR and ${ }^{13} \mathrm{C}$ NMR, respectively. C-2 was observed at $\delta$ 101.25. Strong correlations were observed between $\mathrm{H}-3$ and $\mathrm{H}-4$ in the COSY spectrum. Flav-2-enes did not contain chiral carbons; therefore, optical activity is not observed.

The efficient production of protected 3-oxocatechin analogue 15 (Scheme 4) with the retention of configuration at C-2 by Dess-Martin periodinane oxidation provided a novel approach towards nucleophilic attack at C-3. In contrast to tetrahedral $\mathrm{sp}^{3}$-functionalized C-3 of flavan-3-ol, the $\mathrm{sp}^{2}$-functionalized 3-oxo-group of $\mathbf{1 5}$ was planar, which decreased steric effects and permitted nucleophilic attack on the carbonyl carbon from either the $\alpha$ - or the $\beta$-face. Under this premise, the $\mathrm{C}-\mathrm{C}$ coupling of a nucleophile to the $\mathrm{C}$ 3 of 15 with various Lewis acids (e.g., $\mathrm{AgBF}_{4}, \mathrm{TiCl}_{4}, \mathrm{InCl}_{3}$, and $\left.\mathrm{Yb}(\mathrm{OTf})_{3}\right)$ and solvents $\left(\mathrm{CH}_{2} \mathrm{Cl}_{2}\right.$, THF, DMSO, ethanol, and methanol) was attempted, which failed to yield the expected C-3 coupling products. However, by the treatment of 15 with $\mathrm{Yb}(\mathrm{OTf})_{3}$ in methanol and reflux of the reaction mixture for $6 \mathrm{~h}, 17$ (17\% yield) and 18 (32\% yield) were obtained, probably via a hemiacetal intermediate (3-hydroxy$3,5,7,3^{\prime}, 4^{\prime}$-pentamethoxyflavane) $\mathbf{1 6}$ (Scheme 4). Both $\mathbf{1 7}$ and 18 exhibited a Cotton effect at wavelengths ranging from 260 to $284 \mathrm{~nm}$, indicating no change in the configuration at C2. This observation is in contrast with the racemic products synthesized by Clark-Lewis and Jemison [14].

Enol ether 17 was characterized by the disappearance of the carbonyl resonance in the ${ }^{13} \mathrm{C}$ NMR spectrum of $\mathbf{1 5}$ at $\delta 206.1 \mathrm{ppm}$ and the appearance of an additional methoxy resonance at $\delta 3.73 \mathrm{ppm}$ in the ${ }^{1} \mathrm{H}$ NMR spectrum. The two enol carbons C-2 and C-3 were observed at 77.2 and 150.7, respectively. The two benzylic protons at C-4 of 15 disappeared and were replaced by an olefinic resonance at $\delta 5.60 \mathrm{ppm}$. Structural elucidation is further supported by mass spectrometry $\left(\mathrm{M}^{+}\right.$ion at $\left.m / z 358\right)$, corresponding to the addition of a methyl group and the loss of a hydrogen. A negative Cotton effect in the $\lambda=216 \mathrm{~nm}$ range was observed in the CD spectrum suggesting that optical activity at C-2 (2R-configuration) of the commercial catechin was maintained. This observation was supported by proton NMR coupling constants and the NOESY correction of the $\mathrm{H}-2$ with H-2' and H- ${ }^{\prime}$. The H-4 demonstrated NOESY correlations with the methoxy group on C-3 (Scheme 5).

Ketal 18 was characterized by the disappearance of the carbonyl resonance of $\mathbf{1 5}$ at approximately $\delta 206.1 \mathrm{ppm}$ and the appearance of two additional methoxy resonances at $\delta$ 3.34 and 3.31, respectively. Ketal carbon (C-3) was observed at $\delta 101.2 \mathrm{ppm}$. This result was further supported by the $\mathrm{M}^{+}$ ion at $m / z 391$, corresponding to the addition of two methoxy groups (and the loss of an OH group). NOESY demonstrated correlations between the two benzylic C-4 protons ( $4 \alpha$ and $4 \beta$ ) and the $\mathrm{A}$ ring and C-2-proton. NOESY correlations

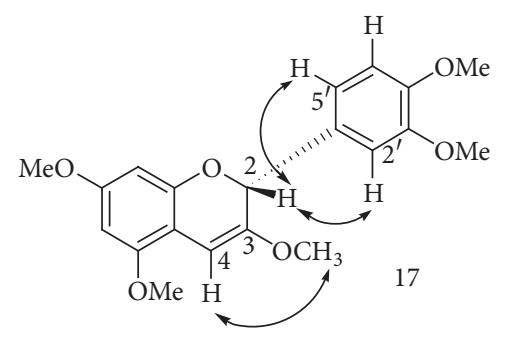

SCheme 5: Observed NOESY interactions between $\mathrm{H}-2$ and $\mathrm{H}-2^{\prime}$ and $\mathrm{H}-6^{\prime}$ for 17.

between $\mathrm{H}-4 \beta$ and $\mathrm{H}-2$, as well as with $\mathrm{H}-2^{\prime}$ and $\mathrm{H}-6^{\prime}$, were observed. The two methoxy groups on C-3 crowded the Cring, and line broadening was observed in the ${ }^{1} \mathrm{H}$ NMR spectrum. This result possibly indicated that conformational correlations exist between $\mathrm{H} \alpha-4$ and $\mathrm{H}-2^{\prime} / \mathrm{H}-6^{\prime}$ and that a significant population of the A conformer [25] (B ring in the axial position) is present. A positive Cotton effect was observed at $\lambda=239 \mathrm{~nm}$.

The condensation of methylated catechin 15a or benzylated catechin $\mathbf{1 5 b}$ with phloroglucinol in the presence of excess $\mathrm{AgBF}_{4}$ in dry THF afforded C-4 coupled product 19a,b (Scheme 6). The carbonyl signals for 19a,b were absent at the expected $\delta$ 204-206 ppm, probably because of a formation of hydrogen bonding between the D-ring neighbouring hydroxyl proton and the carbonyl oxygen. The debenzylation of $\mathbf{1 9 b}$ by hydrogenation afforded 21 with a free phenolic group in good yield. Flav-3-en-3-ols are versatile precursors for flavonoids [26], which play vital roles in the biogenesis pathway of tannins [27]. The acetylation of 19a and 21 afforded 20 and 22, respectively, in good yield. Compound 20 was characterized by the absence of the carbonyl resonance at $\delta 206.3 \mathrm{ppm}$ in the ${ }^{13} \mathrm{C}$ NMR spectrum of the precursor 19a and the presence of four acetoxy resonances at $\delta 1.70,1.95,2.06$, and $2.30 \mathrm{ppm}$, respectively, in the ${ }^{1} \mathrm{H}$ NMR spectrum of 20 . The C-4 proton of $19 a$ at $5.10 \mathrm{ppm}$ disappeared, and the olefinic group at C-3, stabilized 20, was observed. The structure of $\mathbf{2 0}$ conformed to the $\mathrm{M}^{+}$ ion at $m / z$ 636. Octa-acetoxy 22 was characterized by eight acetate groups with proton resonances at $\delta 1.61,1.66,1.82$, $1.99,2.18,2.20,2.21$, and $2.22 \mathrm{ppm}$, respectively, and the ${ }^{13} \mathrm{C}$ NMR resonances were observed at $\delta$ 20.0-21.1 ppm, with the expected $\mathrm{M}^{+}$ion at $m / z 748$. NOESY correction of $\mathrm{H}-2$ with $\mathrm{H}-2^{\prime}$ and $\mathrm{H}-6^{\prime}$ was observed. The $\mathrm{H}-3$ and $\mathrm{H}-4$ protons signals were not observed on the NMR spectra for $\mathbf{2 0}, \mathbf{2 1}$, and 22 compounds. The $2 R$-configuration of the compounds was assessed via coupling constants from their respective spectral data and NMR NOESY proton correlations (Supp. Figure 1 in Supplementary Material available online at https:// doi.org/10.1155/2017/3971253).

Scheme 7 shows the proposed mechanism of the reaction pathway to the oxidative formation of $19 \mathbf{a}, \mathbf{b}$.

The exclusive formation of $\mathbf{1 9} \mathbf{a}, \mathbf{b}$ was primarily attributed to absolute configuration on C-2 $(2 R)$, which predominantly directed the approaching nucleophile to the anti-face $(4 S)$. 


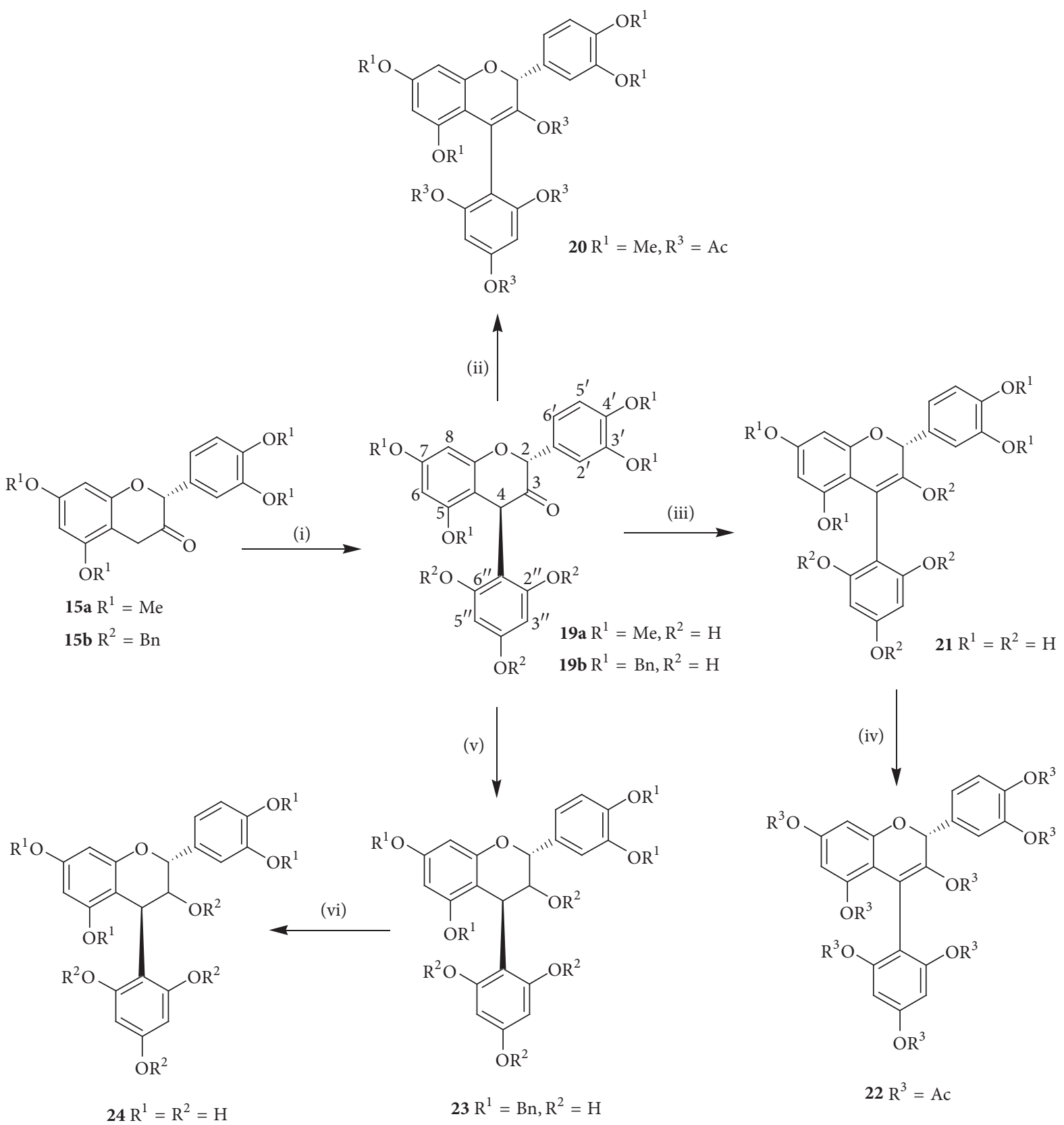

SCHEME 6: Synthesis of 4-substituted flav-3-en-3-o-R substituent. Reagents and conditions: (i) Phlorog., $\mathrm{AgBF}_{4}$, THF, reflux, 61\% (19a), 59\% (19b); (ii) 19a, $\mathrm{Py},\left(\mathrm{CH}_{3} \mathrm{CO}\right)_{2} \mathrm{O}, 40^{\circ} \mathrm{C}, 92 \%$; (iii) 19b, $\mathrm{Pd}(\mathrm{OH})_{2}, \mathrm{H}_{2}$, EtOAc/MeOH, rt, 95\%; (iv) $\mathrm{Py},\left(\mathrm{CH}_{3} \mathrm{CO}\right)_{2} \mathrm{O}, 40^{\circ} \mathrm{C}, 90 \%$; (v) 19b, THF, $\mathrm{NaOH}_{\mathrm{aq}} / \mathrm{EtOH}, \mathrm{NaBH}_{4}, \mathrm{rt}, 99 \%$; (vi) $\mathrm{Pd}(\mathrm{OH})_{2} / \mathrm{C}, \mathrm{H}_{2}$, EtOAc/MeOH, rt, $94 \%$.

Notably, self-condensation products were not observed probably because of the deactivation of the nucleophilic properties of the A ring via the enolic C-ring tautomer (Scheme 7) [22].

\section{Conclusion}

Few synthetic methods have been published to efficiently synthesize flavenes, although none are stereoselective.
Substituted flav-3-enes were exclusively synthesized efficiently by the stereoselective elimination of the 3-Osubstituent on protected catechin using DBU. 3-Substituted flav-3-ene derivatives were prepared by treating flavan-3one with a strong Lewis acid in the presence of a suitable nucleophile. Solvent choice was critical to the success of reactions and yields of corresponding products. Optical activities of the compounds were confirmed by $\mathrm{CD}$ data and NMR spectroscopy analysis. 
<smiles>[R20]c1cc([R20])c2c(c1)O[C@H](c1ccc([14CH3])c([R20])c1)C(=O)C2</smiles>

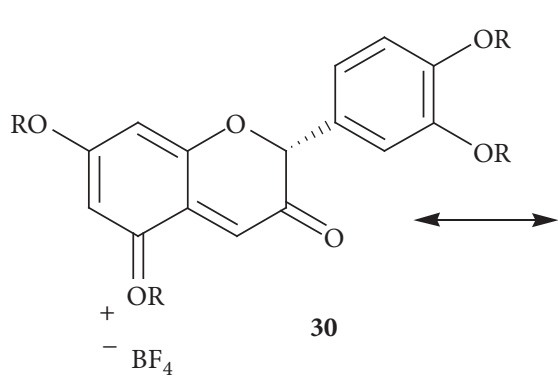

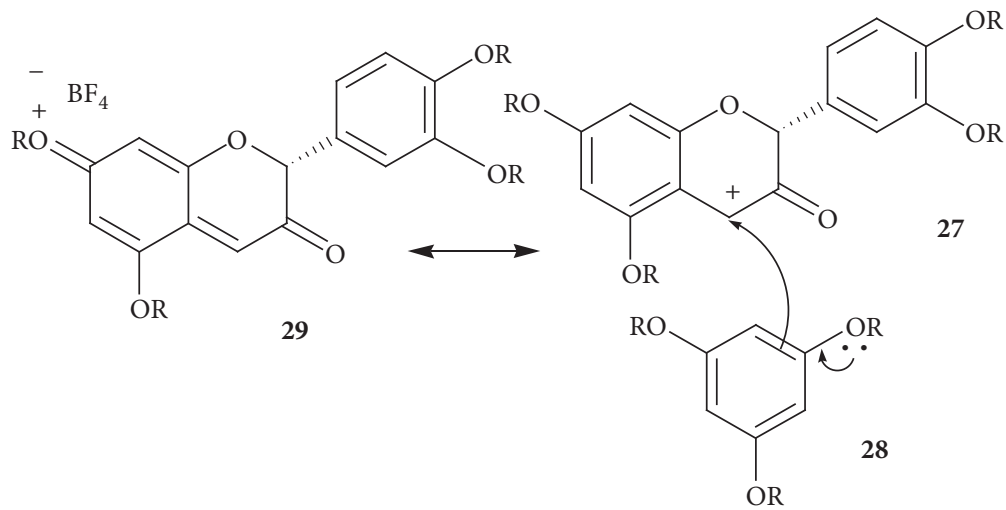<smiles>[R20]OC1=CC(=O)C(C2C(=O)[C@H](c3ccc([R20])c(O[R2])c3)Oc3cc([R6])cc(O)c32)C([R20])=C1</smiles>

SCHeme 7: Proposed mechanism for the formation of 19.

\section{Conflicts of Interest}

The authors declare that they have no conflicts of interest.

\section{Acknowledgments}

Matthew Chilaka Achilonu acknowledges the financial support from the Research Innovation Fund (3513/DHET) of the Central University of Technology Free State, as well as the support of the Chemistry Department of the University of the Free State during his doctoral studies.

\section{References}

[1] H. L. Min, T. C. Eui, M. L. Yoon, D. Kim, and S. P. Yong, "Stereoselective syntheses of 3-substituted flavene derivatives and asymmetric dehydration via kinetic resolution," Bulletin of the Korean Chemical Society, vol. 29, no. 7, pp. 1299-1300, 2008.
[2] C.-W. Lee, H.-J. Choi, H.-S. Kim et al., "Biflavonoids isolated from Selaginella tamariscina regulate the expression of matrix metalloproteinase in human skin fibroblasts," Bioorganic \& Medicinal Chemistry, vol. 16, pp. 732-738, 2008.

[3] X. Zheng, W.-D. Meng, and F.-L. Qing, "Synthesis of gemdifluoromethylenated biflavonoid via the Suzuki coupling reaction," Tetrahedron Letters, vol. 45, no. 43, pp. 8083-8085, 2004.

[4] B. Gil, M. J. Sanz, M. C. Terencio, R. Gunasegaran, M. Paya, and M. J. Alcaraz, "Morelloflavone, a novel biflavonoid inhibitor of human secretory phospholipase A, with anti-inflammatory activity," Biochemical Pharmacology, vol. 53, pp. 733-740, 1997.

[5] M. K. Lee, S. W. Lim, H. Yang et al., "Osteoblast differentiation stimulating activity of biflavonoids from Cephalotaxus koreana," Bioorganic \& Medicinal Chemistry Letters, vol. 16, no. 11, pp. 2850-2854, 2006.

[6] J. Chen, H. W. Chang, H. P. Kim, and H. Park, "Synthesis of phospholipase $\mathrm{A}_{2}$ inhibitory biflavonoids," Bioorganic \& Medicinal Chemistry Letters, vol. 16, no. 9, pp. 2373-2375, 2006. 
[7] B. C. Das, S. Mohapatra, P. D. Campbell, S. Nayak, S. M. Mahalingam, and T. Evans, "Synthesis of function-oriented 2phenyl-2H-chromene derivatives using 1-pipecolinic acid and substituted guanidine organocatalysts," Tetrahedron Letters, vol. 51, no. 19, pp. 2567-2570, 2010.

[8] M. C. Achilonu and D. O. Umesiobi, "The formation of carbon-carbon and carbon-heteroatom bonds using silver tetrafluoroborate as a promoter," Arabian Journal of Chemistry, vol. 9, supplement 2, pp. S1984-S2003, 2016.

[9] M. C. Achilonu and D. O. Umesiobi, "Bioactive phytochemicals: bioactivity, sources, preparations, and/or modifications via silver tetrafluoroborate mediation," Journal of Chemistry, vol. 2015, Article ID 629085, 22 pages, 2015.

[10] S. Guillotin, P. Sanoner, and C. M. G. C. Renard, "Stabilisation of the colour of anthocyanins in solutions by admixture with phytocomponents from apple," Journal of Horticultural Science \& Biotechnology ISAFRUIT Special Issue, pp. 96-99, 2009.

[11] J. W. Clark-Lewis and M. I. Baig, "Flavan derivatives. XXXII. Structures and N.M.R. spectra of flav-2-enes and flav-3-enes; hydroboration and hydroxylation of flav-3-enes," Australian Journal of Chemistry, vol. 24, no. 12, pp. 2581-2592, 1971.

[12] G. A. Iacobucci and J. G. Sweeny, "The chemistry of anthocyanins, anthocyanidins and related flavylium salts," Tetrahedron, vol. 39, no. 19, pp. 3005-3038, 1983.

[13] L. Jurd, "Structural and Functional Aspects of Phytochemistry," in Recent progress in Chemistry of Flavylium Salts, V. C. Rumeckles and T. C. Tso, Eds., vol. 5, pp. 135-142, Academic Press, New York, NY, USA, 1972.

[14] J. W. Clark-Lewis and R. W. Jemison, "Flavan derivatives. XXVIII. Stereochemistry of products synthesized from flav-3enes and flavanones," Australian Journal of Chemistry, vol. 23, no. 2, pp. 315-321, 1970.

[15] N. T. Zaveri, "Synthesis of a 3,4,5-trimethoxybenzoyl ester analogue of epigallocatechin-3-gallate (EGCG): a potential route to the natural product green tea catechin, EGCG," Organic Letters, vol. 3, no. 6, pp. 843-845, 2001.

[16] A. Pelter and P. Stainton, "The mass spectra of oxygen heterocycles. Part (V). The mass spectra of $2^{\prime}$-hydroxyflavonoids," Journal of the Chemical Society C: Organic Chemistry, pp. 19331937, 1967.

[17] B. Nay, V. Arnaudinaud, J.-F. Peyrat et al., "Total synthesis of isotopically labelled flavonoids, $2:{ }^{13} \mathrm{C}$-labelled ( \pm )-catechin from potassium $\left[{ }^{13} \mathrm{C}\right]$ cyanide," European Journal of Organic Chemistry, no. 7, pp. 1279-1283, 2000.

[18] M. Deodhar, D. S. Black, and N. Kumar, "Acid catalyzed stereoselective rearrangement and dimerization of flavenes: synthesis of dependensin," Tetrahedron, vol. 63, no. 24, pp. 52275235, 2007.

[19] G. Casiraghi and G. Casnati, "A new method for the synthesis of flav-2-enes," Journal of the Chemical Society D: Chemical Communications, vol. 5, p. 321, 1970.

[20] G. Cardillo, R. Cricchio, and L. Merlini, "Biogenetic-like synthesis of flav-3-enes from o-cinnamylphenols," Tetrahedron Letters, vol. 10, no. 12, pp. 907-908, 1969.

[21] R. S. Subramanian and K. K. Balasubramanian, "A novel synthesis of flav-3-enes by claisen rearrangement," Tetrahedron Letters, vol. 29, no. 51, pp. 6797-6799, 1988.

[22] M. C. Achilonu, S. L. Bonnet, and J. H. van der Westhuizen, "Synthesis of proanthocyanidins. Part 1. The first oxidative formation of the interflavanyl bond in procyanidins," Organic Letters, vol. 10, no. 17, pp. 3865-3868, 2008.
[23] A. P. Kozikowski, W. Tückmantel, and C. George, "Studies in polyphenol chemistry and bioactivity. 2. Establishment of interflavan linkage regio- and stereochemistry by oxidative degradation of an $O$-alkylated derivative of procyanidin B2 to (R)-(-)-2,4-diphenylbutyric acid," Journal of Organic Chemistry, vol. 65, no. 17, pp. 5371-5381, 2000.

[24] J. F. O’Connell and H. Rapoport, "1-Benzenesulfonyl- and 1-p-toluenesulfonyl-3-methylimidazolium triflates: efficient reagents for the preparation of arylsulfonamides and arylsulfonates," Journal of Organic Chemistry, vol. 57, no. 17, pp. 4775-4777, 1992.

[25] P. J. Steynberg, J. P. Steynberg, B. C. B. Bezuidenhoudt, and D. Ferreira, "Oligomeric flavanoids. Part 19. Reductive cleavage of the interflavanyl bond in proanthocyanidins," Journal of the Chemical Society, Perkin Transactions 1, no. 23, pp. 3005-3012, 1995.

[26] D. Jacques, C. T. Opie, L. J. Porter, and E. Haslam, "Plant proanthocyanidins. Part 4. Biosynthesis of procyanidins and observations on the metabolism of cyanidin in plants," Journal of the Chemical Society, Perkin Transactions 1, no. 14, pp. 1637$1643,1977$.

[27] J. Coetzee, E. Malan, and D. Ferreira, "Synthesis and reactions of flav-3-en-3-ols," Tetrahedron, vol. 56, no. 13, pp. 1819-1824, 2000 . 

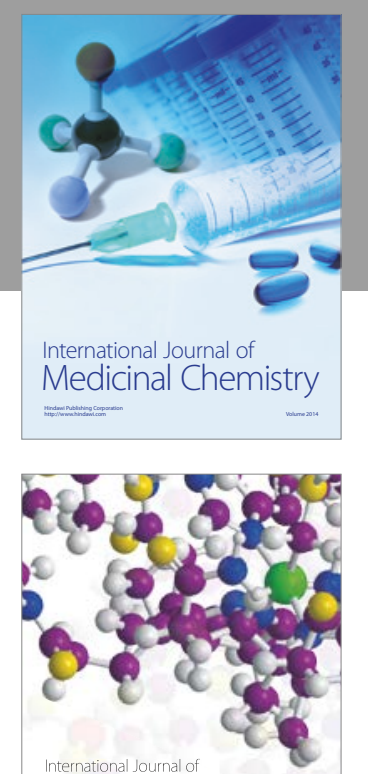

Carbohydrate Chemistry

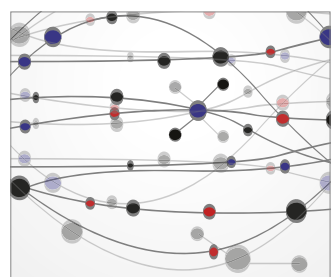

The Scientific World Journal
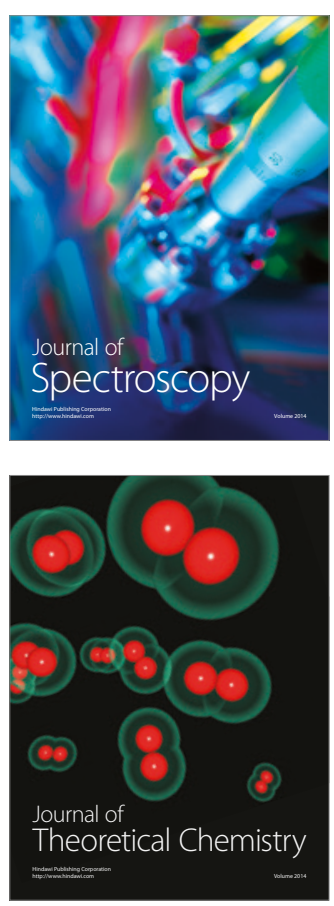
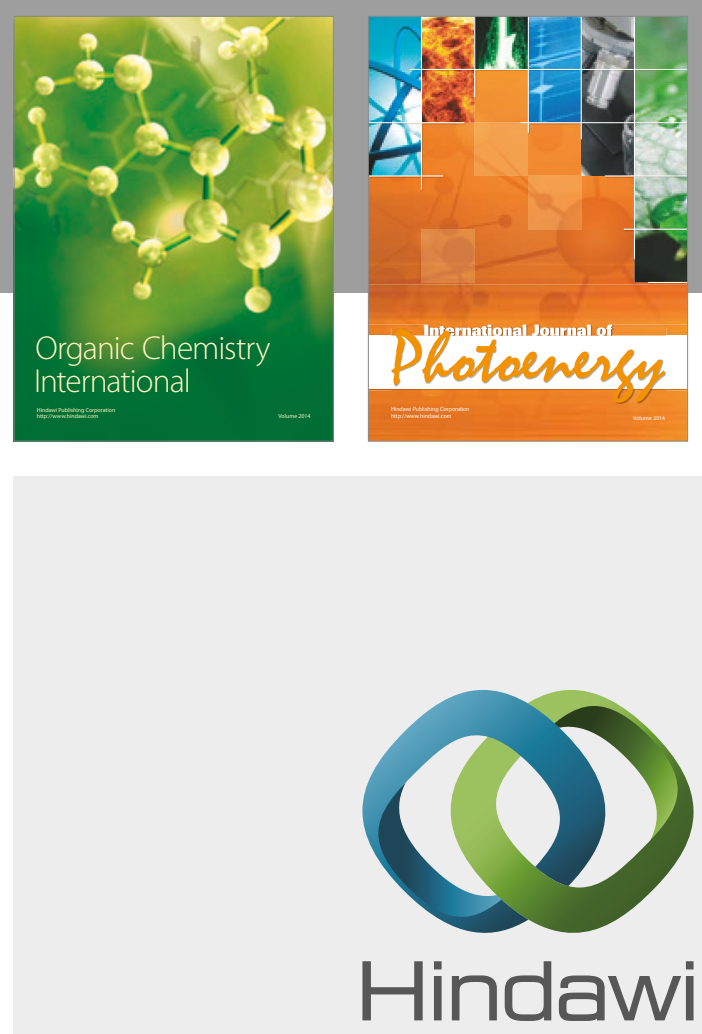

Submit your manuscripts at

https://www.hindawi.com

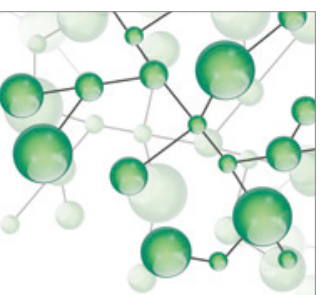

International Journal of

Inorganic Chemistry

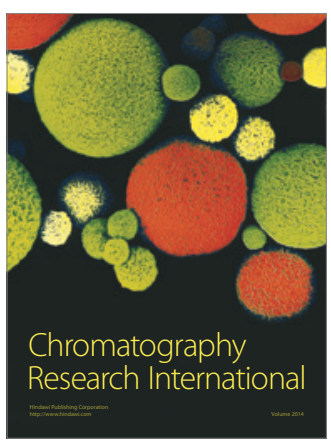

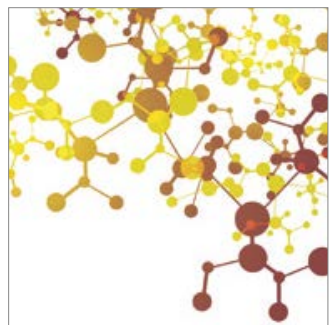

Applied Chemistry
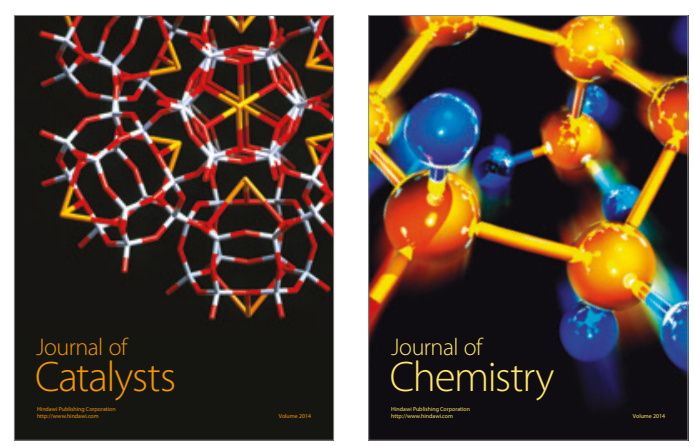
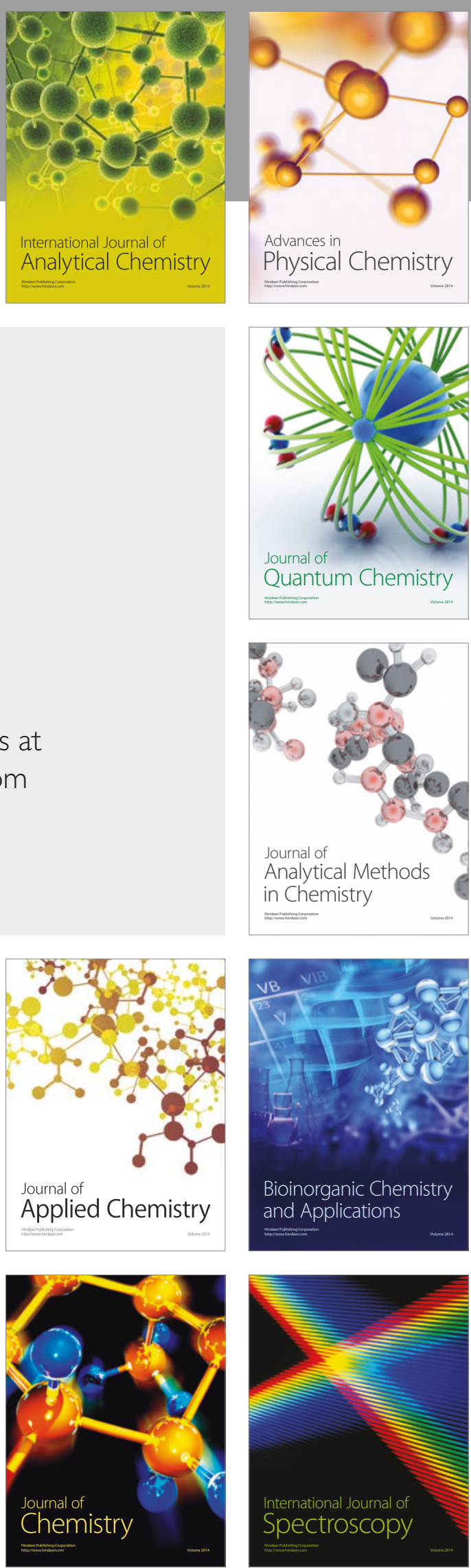